\title{
Large-spin asymptotics of Euclidean LQG flat-space
}

\section{wavefunctions}

\author{
Aleksandar Miković $\dot{c}^{1,2}$ and Marko Vojinović ${ }^{2}$
}

${ }^{1}$ Departamento de Matemática, Universidade Lusófona de Humanidades e

Tecnologia, Av. do Campo Grande, 376, 1749-024, Lisboa, Portugal amikovic@ulusofona.pt

${ }^{2}$ Grupo de Física Matemática da Universidade de Lisboa, Av. Prof. Gama Pinto, 2, 1649-003 Lisboa, Portugal

vmarko@cii.fc.ul.pt

\begin{abstract}
We analyze the large-spin asymptotics of a class of spin-network wavefunctions of Euclidean loop quantum gravity, which corresponds to a flat spacetime. A wavefunction from this class can be represented as a sum over the spins of an amplitude for a spin network whose graph is a composition of the wavefunction spin network graph with the dual one-complex graph and the tetrahedron graphs for a triangulation of the spatial 3-manifold. This spin-network amplitude can be represented as a product of $6 j$ symbols, which is then used to find the large-spin asymptotics of the wavefunction. By using the Laplace method we show that the large-spin asymptotics is given by a sum of Gaussian functions. However, these Gaussian functions are not of the type, which gives the correct graviton propagator.
\end{abstract}

e-print archive: http://lanl.arXiv.org/abs/1005.1866v1 


\section{Introduction}

Loop quantum gravity (LQG) is a theory of non-perturbative and background-independent quantization of GR; see [1]. It is based on the canonical quantization method, and instead of spatial metric, a spatial connection is used as the configuration space variable. Consequently, the Hilbert space of physical states is spanned by the spin network states $|\Gamma\rangle$, where $\Gamma$ is a closed $S U(2)$ spin network. The graph of $\Gamma$ is a combinatorial graph, i.e., it is a homotopy class, because the spin-network states are diffeomorphism invariant. A physical state $|\Psi\rangle$ is given as a linear combination of the spinnetwork states such that it satisfies the quantum Hamiltonian constraint $\mathcal{H}|\Psi\rangle=0$, where $\mathcal{H}$ is the Hamiltonian constraint operator.

Solving the Hamiltonian constraint is a difficult problem, and various strategies have been developed over the years. In particular, one can consider the quantum Hamiltonian constraint in the Ashtekar connection representation, where $\mathcal{H}$ becomes a polynomial in the functional derivatives. Then any functional $\Psi(A)$ with a support on flat connections is a solution when the cosmological constant is zero; see [2]. The Ashtekar connection is complex in the Minkowski signature case, so that the resolution of the identity is given by

$$
\int \mathcal{D}(\operatorname{Re} A) \mathcal{D}(\operatorname{Im} A)|A\rangle\langle A|=I
$$

since the operator $A$ is similar to the annihilation operator for the harmonic oscillator. The resolution of the identity (1.1) is an obstacle to construct the loop transform from $\Psi(A)$ to the spin network wavefunction $\Psi(\Gamma)$. However, in the Euclidean signature case, the Ashtekar connection is real, so that one obtains the usual expression

$$
\int \mathcal{D} A|A\rangle\langle A|=I
$$

Consequently

$$
\langle\Gamma \mid \Psi\rangle=\int \mathcal{D} A \overline{W_{\Gamma}(A)} \Psi(A)
$$

where $W_{\Gamma}(A)=\langle A \mid \Gamma\rangle$ is the generalization of the trace of the holonomy along a curve to a spin network; see [1].

The path integral (1.2) can be rigorously defined by using a three-dimensional spin-foam state sum for the quantum group $U_{q}(s u(2))$ where 
$q=\exp \left(\frac{\mathrm{i} \pi}{k+2}\right)$ is a root of unity $[2,3]$. When $\Psi(A)=$ const $\cdot \delta(F)$ then (1.2) becomes an invariant $\Psi_{k}(\Gamma)$, which is proportional to the WittenReshetikhin-Turaev invariant for a spin network $\Gamma$ embedded in a compact 3 -manifold $\Sigma$ representing a spatial slice of the spacetime [4]. This means that the state

$$
\left|\Psi_{k}\right\rangle=\sum_{\Gamma} \Psi_{k}(\Gamma)|\Gamma\rangle
$$

corresponds to the Kodama wavefunction

$$
\Psi_{k}(A)=\exp \left(\mathrm{i} \frac{k}{4 \pi} \int_{\Sigma} \operatorname{Tr}\left(A \wedge d A+\frac{2}{3} A \wedge A \wedge A\right)\right) .
$$

Therefore, the effect of using a quantum $S U(2)$ group is that the flatconnection state, represented by the wavefunction $\Psi(A)$, is deformed into the Kodama state (1.3). This state is a physical state for quantum GR with the cosmological constant $\Lambda_{k}$ proportional to $1 / k$, where $k$ is an integer.

When $\Psi(A)=\exp \left(\mathrm{i} \int_{\Sigma} \operatorname{Tr}\left(E^{m} A_{m}\right)\right) \delta(F)$, it can be argued that the corresponding quantum group state $\left|\Psi_{k}\right\rangle$ is a physical state for quantum GR with the cosmological constant $\Lambda_{k}$ such that the triads take the values $E_{m}$; see [3]. Hence the spin-network wavefunction $\Psi_{k}(\Gamma, E)$ gives a physical state describing a spacetime whose spatial metric is given by $g_{m n}=\operatorname{Tr}\left(E_{m} E_{n}\right)$. In particular, one can choose the flat triads and hence $\left|\Psi_{k}\right\rangle$ can be considered as a Euclidean analog of the vacuum state for a De Sitter spacetime. Since $\Lambda_{k} \rightarrow 0$ as $k \rightarrow \infty$, then $\left|\Psi_{k}\right\rangle$ for large $k$ can be considered as a good approximation for the Euclidean flat-spacetime vacuum.

This result is very useful for the problem of finding a semiclassical limit of LQG, since it can be shown that the graviton propagator in LQG will have the correct large-distance asymptotics if the vacuum wavefunction $\Psi(\Gamma, E)$ has a certain kind of Gaussian asymptotics when the spins of $\Gamma$ are large [5]; see also $[6,7]$ for a related approach.

As shown in [5], if the large-spin asymptotics is given by

$$
\Psi\left(\Gamma, j_{0}\right) \approx N\left(\Gamma, j_{0}\right) \exp \left(-\sum_{l, l^{\prime}} \frac{C_{l l^{\prime}}(\Gamma)}{j_{0}}\left(j_{l}-j_{0}\right)\left(j_{l^{\prime}}-j_{0}\right)\right),
$$

where $j_{l}$ is a spin of an edge $l, j_{0}$ is a parameter associated to the flat spatial metric and $C(\Gamma)$ are $j_{0}$-independent and positive-definite matrices, then 
the corresponding graviton propagator will have the correct large-distance asymptotics.

In order to analyze the large-spin asymptotics of $\Psi_{k}\left(\Gamma, j_{0}\right)$, we will represent it as a sum over spins of products of quantum dimensions and quantum $6 j$ symbols. When $k$ is very large, we will approximate $\Psi_{k}$ by the corresponding Ponzano-Regge expression, i.e., we will replace the quantum dimensions and the quantum $6 j$ symbols in the expression for $\Psi_{k}$ with the corresponding classical evaluations. The rationale for this is that $\Psi_{k}\left(\Gamma, j_{0}\right)$ was constructed as a regularization of an expression for the zero-cosmological constant $\Psi\left(\Gamma, j_{0}\right)$, which was given by the Ponzano-Regge state-sum without the spin cut-off; see [3]. If a spin cut-off $k / 2$ is introduced, one obtains another regularization of $\Psi\left(\Gamma, j_{0}\right)$, such that the quantum group is not used. This was the regularization originally used by Ponzano and Regge. Furthermore, the sums $\sum_{0}^{k / 2}$ in $\Psi_{k}\left(\Gamma, j_{0}\right)$ will be replaced by the integrals $\int_{0}^{k / 2}$, since $k$ is very large. Consequently

$$
\Psi\left(j, j_{0}\right) \approx \int_{D} d^{\omega} x f\left(x, j, j_{0}\right) .
$$

We will split the integration region $D$ as $D=D_{-} \cup D_{+}$where $D_{+}$is the region where all the spins are large $\left(x_{i}>j_{0}\right)$, so that $\Psi \approx \Psi_{-}+\Psi_{+}$. Consequently the $6 j$ symbols from $\Psi_{+}$can be approximated by the PonzanoRegge formula, while the $6 j$ symbols from $\Psi_{-}$can be approximated by the asymptotic formulas for 5, 4 and 3 large spins, which were also found by Ponzano and Regge; see [9]. We will study in detail the asymptotics of $\Psi_{+}$, while the analysis of $\Psi_{-}$will be only sketched since it is very similar to the analysis of $\Psi_{+}$and it can be shown that the $\Psi_{-}$asymptotic contribution is subleading to that of $\Psi_{+}$.

We will use the Laplace method to find the asymptotics of (1.5) for large $j$ and $j_{0}$. In order to do this we will first approximate $f$ as a sum of exponentials; see Section 3. Then it is not difficult to show that

$$
\Psi\left(j, j_{0}\right) \approx \sum_{n, \pm} N_{n}^{ \pm}\left(j_{0}\right) \mathrm{e}^{-1 / 2\left(j-j_{0}\right)^{\mathrm{T}}\left(B_{n}^{ \pm}+\frac{1}{j_{0}} C_{n}^{ \pm}+O\left(1 / j_{0}^{2}\right)\right)\left(j-j_{0}\right)},
$$

where $B_{n}^{ \pm}$e $C_{n}^{ \pm}$are constant ( $j_{0}$-independent) matrices.

The expression (1.6) will give the desired asymptotics if $B_{n}^{ \pm}=0$ for all $n$ and all spin networks and $C_{n}^{ \pm} \neq 0$ for some $n$ of some spin network. In the following sections, we will show that either $B_{n}^{+} \neq 0$ or $C_{n}^{+}=0$ for all $n$ and all spin networks. We will also show that $C_{n}^{-}=0$ for all $n$ and all 
spin networks, so that we will prove that $\Psi\left(j, j_{0}\right)$ does not have the desired asymptotics.

The paper is organized in the following way. In Section 2, we briefly explain the construction of the relevant spin-network wavefunctions from [2-4]. In Section 3, we outline the main procedure of analyzing the wavefunction asymptotics, and introduce the notation. Section 4 deals with the detailed analysis of the large-spin asymptotics of the $\Psi_{+}$part of the wavefunction. The $\Psi_{+}$is written as an integral of an exponential function, which is suitable for the stationary-point approximation. The integration is then performed in Section 5 and the result is a sum of Gaussian functions of the form similar to (1.4), but with a more general matrix coefficient in the exponent, denoted as $\tilde{S}$. In Section 6 , we analyze this coefficient, and prove that it never has the form (1.4). In order to demonstrate and verify this result further, in section 7 the matrix $\tilde{S}$ is explicitly computed for two simple spin-networks, which are a loop spin network and a theta spin network. In Section 8 , we discuss the large-spin asymptotics of the $\Psi_{-}$part of the wavefunction. We present our conclusions in 9, while in the appendix we give all the necessary formulas and prove a matrix theorem which determines the asymptotics of the $\tilde{S}$ matrix.

\section{Physical spin-network wavefunctions}

Let $\Sigma$ be a compact 3-manifold, and let $\Gamma=\left\{\gamma, j_{l}, \iota_{v}\right\}$ be a spin network embedded in $\Sigma$, where $\gamma$ is the spin-network graph, $j_{l}$ denote the edge spins and $\iota_{v}$ denote the vertex intertwiners. Let $\Delta(\Sigma)$ be a triangulation of $\Sigma$ adapted to $\gamma$ in the following way: let $H$ be a handle-body obtained by thickening of the dual one-complex $\Delta_{1}^{*}(\Sigma)$. The graph $\gamma$ is embedded in $H$ such that each vertex $v_{\gamma}$ is placed in a different 3-handle of $H$ and each edge $l_{\gamma}$ runs through appropriate one-handles of $H$; see figure 1 . Let $L_{H}$ be the Chain-Mail link associated with $H$ and let $L_{1}$ be a set of loops associated to the edges of $\gamma$, such that $L_{1}$ is embedded in the one-handle of $H$ associated to an edge $l$ and $L_{1}$ is linked with the meridian loop for that one-handle; see figure 2 .

Let $\phi: \Sigma \rightarrow S^{3}$ be a smooth map from $\Sigma$ to a 3 -sphere, and let us color the link $L_{H}$ with the $\Omega$ elements. The $\Omega$ element is a linear combination of colors given by

$$
\Omega=\sum_{j=0}^{k / 2} \operatorname{dim}_{q} j C(j),
$$




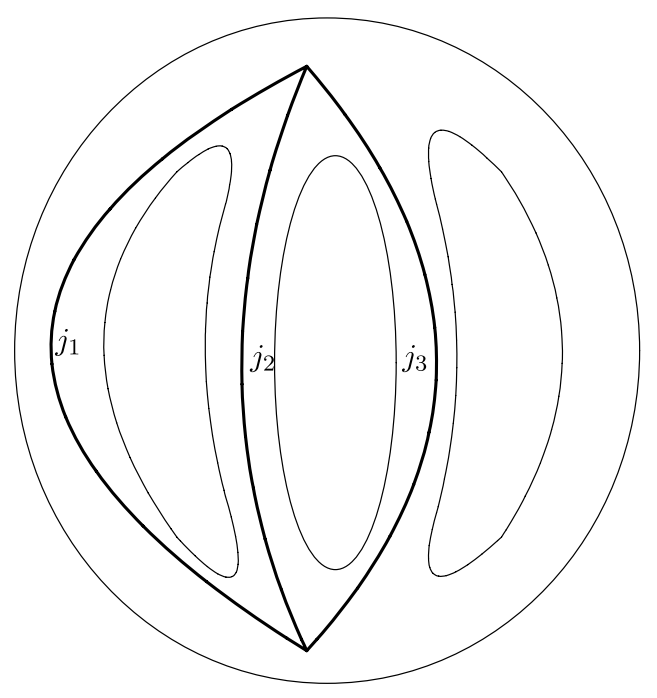

Figure 1: A theta spin network embedded into a handlebody for a 3 -sphere.

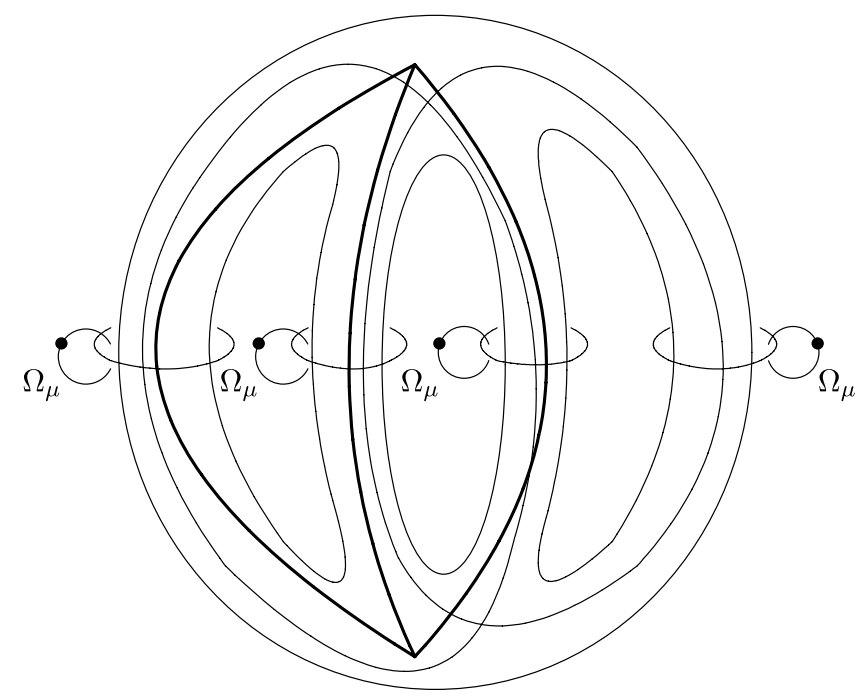

Figure 2: The Omega link for the spin network and the handlebody from Fig. 1.

where $C(j)$ denotes the color $\left(\operatorname{spin} j\right.$ ) associated to a loop of $L_{H}$. We also introduce

$$
\Omega_{\mu}=\sum_{j=0}^{k / 2} \mu(j) C(j) .
$$


Let us color the $L_{1}$ loops with $U_{q}(s u(2))$ irreps $\lambda_{l}$. We will denote the quantum group evaluation of the colored link $L_{H} \cup L_{1} \cup \cdots \cup L_{n} \cup \gamma$ embedded in $S^{3}$ as

$$
\left\langle L_{H} \cup L_{1} \cup \cdots \cup L_{n_{2}} \cup \gamma, \Omega^{n_{2}+n_{3}}, j, \iota, \lambda\right\rangle,
$$

where $n_{2}$ is the number of dual edges (triangles) and $n_{3}$ is the number of dual vertices (tetrahedrons).

From the properties of the $\Omega$ element (see [4]) it follows that the evaluation (2.1) can be expressed as

$$
\sum_{j^{\prime}, \iota^{\prime}} \prod_{f} \operatorname{dim}_{q} j_{f}^{\prime}\left\langle\Delta_{1}^{*} \cdot(T e t)^{n_{3}} \cdot \gamma, j^{\prime}, \iota^{\prime}, j, \iota, \lambda\right\rangle,
$$

where $\left\{\Delta_{1}^{*} \cdot(\text { Tet })^{n_{3}} \cdot \gamma, j^{\prime}, \iota^{\prime}, j, \iota, \lambda\right\}$ is the spin network, which is obtained by removing $n_{2} \Omega$-elements from $n_{2}$ 1-handle meridians.

Then

$$
\Psi_{k}(\Gamma, E)=\sum_{\lambda, j^{\prime}, \iota^{\prime}} \prod_{l} \mu\left(\lambda_{l}, E_{l}\right) \prod_{f} \operatorname{dim}_{q} j_{f}^{\prime}\left\langle\Delta_{1}^{*} \cdot(T e t)^{n_{3}} \cdot \gamma, j^{\prime}, \iota^{\prime}, j, \iota, \lambda\right\rangle,
$$

where

$$
\mu(\lambda, E)=\frac{1}{\operatorname{dim} \lambda} \int_{S U(2)} d g f(g, E) \chi^{(\lambda)}(g),
$$

and $\chi_{\lambda}$ is the trace of the $\lambda$-representation matrix of $g$. The function $f(g, E)$ is determined by the choice of the flat-connection wavefunctional given by

$$
\Psi(A)=\exp \left(\mathrm{i} \int_{\Sigma} \operatorname{Tr}\left(E^{m} A_{m}\right)\right) \psi(A) \delta(F) .
$$

This $\Psi(A)$ solves the Hamiltonian constraint for $\Lambda=0$, but if $\psi(A) \neq 1$, then $\Psi(A)$ does not have the sharp values for the tetrads, which is the LQG equivalent of replacing a plane-wave with a coherent state.

One can introduce the background spins $j_{l}^{0}$ associated to the triads $E$, via the relation $\left|E_{l}\right|=j_{l}^{0} L^{2}$, where

$$
E_{l}=\int_{\Delta_{l}} E^{m} \epsilon_{m n p} d x^{n} \wedge d x^{p},
$$

$\Delta_{l}$ is the triangle dual to a dual edge $l$ and $L$ is the Planck length. In the flat-triad case, one can assume that $\left|E_{l}\right|=$ const, and therefore $j_{l}^{0}=j_{0}$, so 
that $\Psi_{k}(\Gamma, E)=\Psi_{k}\left(\Gamma, j_{0}\right)$. We will then choose

$$
\mu(\lambda)=\frac{\mathrm{e}^{-\left(\lambda-j_{0}\right)^{2}}}{2 \lambda+1}
$$

in order to mimic the Rovelli ansatz for the wavefunction [6]. We will then examine the asymptotics of (2.2) for large spins $j$ and $j_{0}$.

We will express the evaluation of the $\Delta_{1}^{*} \cdot(\text { Tet })^{n_{3}} \cdot \gamma$ spin network as a sum of products of quantum $6 j$ symbols, since this will facilitate our analysis of the large-spin asymptotics. This can be done because the evaluation of an arbitrary three-valent spin network $\Gamma$ can be represented as a sum of products of $6 j$ symbols; see $[4,8]$.

Let $\Gamma^{\prime}$ be a projection of $\Gamma$ onto an $S^{2}$. The graph $\gamma^{\prime}$ will divide the sphere into disjoint discs. Color the discs with $S U(2)$ irreps $\alpha_{1}, \ldots, \alpha_{n}$ and write the corresponding Turaev shadow-world evaluation $w(\Gamma, \alpha)$, which is given as a product of $6 j$ symbols. Then

$$
\langle\Gamma\rangle \propto \sum_{\alpha} \prod_{i=1}^{n} \operatorname{dim}_{q} \alpha_{i} w(\Gamma, \alpha)
$$

Alternatively, (2.4) is the quantum group evaluation of the link formed by $\gamma^{\prime}$ and the Chain-Mail link for a two-dimensional handle-body, which is a thickening of $\gamma^{\prime}[4]$.

By using (2.2) and (2.4), we obtain the following expression for the spinnetwork wavefunction

$$
\Psi_{k}\left(j, j_{0}\right)=\sum_{a, \alpha, \lambda, \iota}\left(\prod_{a} d_{a} \prod_{\alpha} d_{\alpha} \prod_{\lambda} \mu(\lambda) \prod_{v}\left\{6 j_{v}\right\}\right)
$$

where

- spins $j$ label the graph $\gamma$,

- spins $a$ label the faces of $\Delta^{*}$;

- spins $\lambda$ label the edges of $\Delta_{1}^{*}$;

- spins $\alpha$ label the disjoint discs of the projected graph $\Delta_{1}^{*} \cdot(T e t)^{n} \cdot \gamma$;

- $\left\{6 j_{v}\right\}$ denotes the $6 j$-symbol associated to a vertex $v$ of the projected graph $\Delta_{1}^{*} \cdot(\text { Tet })^{n_{3}} \cdot \gamma$;

- $d_{x}$ is the quantum dimension of the representation $x$;

- $\iota$ denote the intertwiners for the graph $\Delta_{1}^{*} \cdot(\text { Tet })^{n_{3}} \cdot \gamma$. 


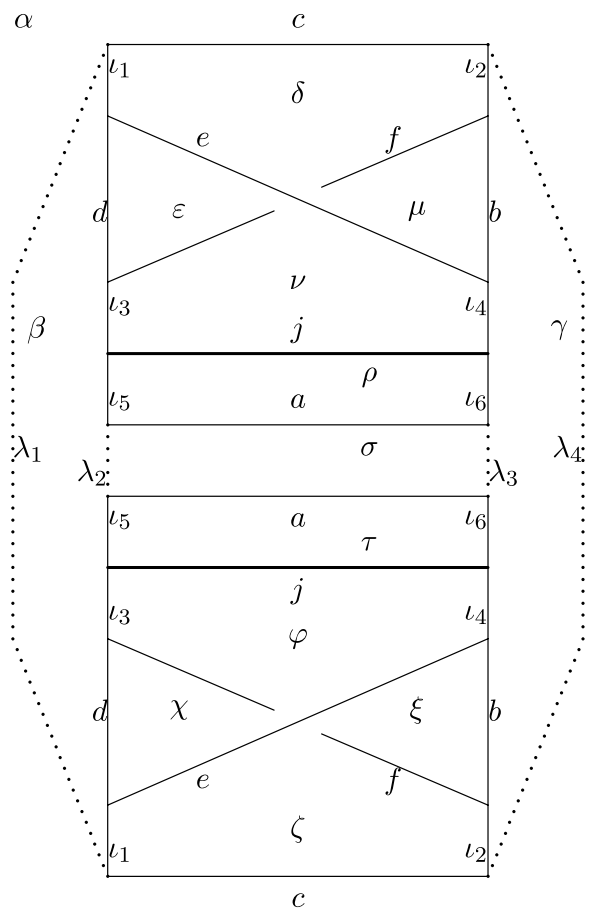

Figure 3: The 3-valent spin network associated to a loop spin network embedded into the handlebody from Fig. 1.

For example, when $\Gamma=\{\gamma, j\}$ where $\gamma$ is a loop embedded in $\Sigma=S^{3}$, we can triangulate $S^{3}$ with two tetrahedrons such that $\Delta_{1}^{*}$ is a theta-four graph $\theta_{4}$. The corresponding 3 -valent $\theta_{4} \cdot(\text { Tet })^{2} \cdot \gamma$ spin network is given in figure 3 in the appendix.

We will also use the Latin indices $M, N, p, q, r, s$ to denote any of the $j, a, \alpha, \lambda$ and $\iota$ indices, and

$$
\begin{gathered}
j \in \mathcal{J}=\{1, \ldots, J\}, \quad a \in \mathcal{A}=\{J+1, \ldots, J+A\}, \\
\alpha, \iota \in \mathcal{Y}=\{J+A+1, \ldots, J+A+\Upsilon\}, \\
\lambda \in \mathcal{L}=\{J+A+\Upsilon+1, \ldots, J+A+\Upsilon+L\}, \\
N \in \mathcal{N}=\{1, \ldots, \Omega\}, \quad \Omega \equiv J+A+\Upsilon+L .
\end{gathered}
$$

The corresponding sums and products will be over the whole domain appropriate for each type of index, unless otherwise noted. We will often have the set of all values of the indices except for the $j$-indices, so we denote it as

$$
\mathcal{I}=\mathcal{N} / \mathcal{J} .
$$

It has $\omega \equiv \Omega-J$ elements. 
The $6 j$ symbols $\left\{6 j_{v}\right\}$ are functions of all spins from a set $J$. The index $v$ will also be treated as a multi-index for spins, in the sense that the $6 j$ symbol

$$
\left\{\begin{array}{lll}
x_{1} & x_{2} & x_{3} \\
x_{4} & x_{5} & x_{6}
\end{array}\right\}
$$

is enumerated by the index $v=(1,2,3,4,5,6)$, representing the ordered 6 -tuple of spins, which appear in that $6 j$ symbol. This will be useful in limiting the domain of indices to a particular $6 j$ symbol, noted as $M, N \in v$.

The function $\mu(\lambda)$ can be chosen arbitrarily, but as we explained in Section 2, we will choose the Gaussian function (2.3). In this way a spin scale, $j_{0}$, is conveniently introduced in the wavefunction.

\section{Preliminary analysis}

As we explained in the introduction, the quantum group expression (2.5) will be replaced by the corresponding classical group expression, where the spins will have a cut-off given by $k / 2$. Since we are interested in the case where $k$ is a very large number, then the finite sums $\sum_{0}^{k / 2}$ in $(2.2)$ can be approximated by the integrals $\int_{0}^{k / 2}$, so that

$$
\Psi\left(j, j_{0}\right) \approx \int_{D} d^{\omega} x f\left(x, j, j_{0}\right)
$$

where

$$
f\left(x, j, j_{0}\right)=\prod_{a}(2 a+1) \prod_{\alpha}(2 \alpha+1) \prod_{\lambda} \frac{\mathrm{e}^{-\left(\lambda-j_{0}\right)^{2}}}{2 \lambda+1} \prod_{v}\left\{6 j_{v}\right\} .
$$

The domain $D$ is a subset of $(k / 2)^{N}$ and $D$ is determined by the triangle conditions for the spins coming from the $6 j$ symbols.

Let us split the integration region $D$ as $D=D_{-} \cup D_{+}$where $D_{+}$is the region, where all the spins are large $\left(x \geq j_{0}\right.$ for every $\left.x\right)$, and $D_{-}=D \backslash D_{+}$. Consequently,

$$
\Psi\left(j, j_{0}\right) \approx \int_{D_{-}} d^{\omega} x f\left(x, j, j_{0}\right)+\int_{D_{+}} d^{\omega} x f\left(x, j, j_{0}\right)=\Psi_{-}\left(j, j_{0}\right)+\Psi_{+}\left(j, j_{0}\right) .
$$

The $6 j$ symbols from $\Psi_{+}$can be approximated by the PR formula, while the $6 j$ symbols from $\Psi_{-}$can be approximated by the asymptotic formulas for 5 , 
4 and 3 large spins. Namely, there will be a certain number of tetrahedrons which contain the large spins $j$, so that each of these tetrahedrons will have at least two other large spins, due to the triangle inequalities. Each of these large spins appear in other tetrahedrons, which will force another spins to be large and so on. In the end there will be a substantial number of large spins different from $j$, but some of the internal spins can still remain small. ${ }^{1}$ Consequently,

$$
\Psi_{ \pm}\left(j, j_{0}\right) \approx \int_{D_{ \pm}} d^{\omega} x f_{ \pm}\left(x, j, j_{0}\right)
$$

where $f_{ \pm}$are the corresponding approximations for $f$ in $D_{ \pm}$regions.

We will use the Laplace method to find the asymptotics of (3.1) for large $j$ and $j_{0}$. In order to do this, we will first approximate $f_{ \pm}$as sums of exponentials. Namely, if $x_{n}$ are the stationary points of $f(x)$ then

$$
f\left(x, j, j_{0}\right) \approx \sum_{n} \epsilon_{n} \mathrm{e}^{-S_{n}\left(x, j, j_{0}\right)},
$$

where $S_{n}\left(x, j, j_{0}\right) \approx|\ln | f\left(x, j, j_{0}\right)||$ in the vicinity of $x_{n}$ and $\epsilon_{n}= \pm 1$ depending on whether $x_{n}$ is a minimum or a maximum. Consequently,

$$
\Psi\left(j, j_{0}\right) \approx \sum_{n} \epsilon_{n} \int_{D} d^{N} x \mathrm{e}^{-S_{n}\left(x, j, j_{0}\right)}=\sum_{n} \epsilon_{n} I_{n}
$$

and one can apply the Laplace method to evaluate the integrals $I_{n}$. This gives

$$
\Psi \approx \sum_{n} \epsilon_{n} \sum_{x^{*}, j^{*}} N_{n}^{*}\left(j_{0}\right) \mathrm{e}^{-\frac{1}{2}\left(j-j_{n}^{*}\right)^{T} \tilde{S}_{n}^{*}\left(j_{0}\right)\left(j-j_{n}^{*}\right)},
$$

where $x^{*}, j^{*}$ are the stationary points of $S$ and

$$
\tilde{S}^{*}=S_{j j}^{*}-\left(S_{x j}^{*}\right)^{T} S_{x x}^{*} S_{x j}^{*},
$$

where $S_{j j}^{*}, S_{x j}^{*}$ and $S_{x x}^{*}$ denote the Hessian matrices in the respective stationary points.

In order to find the matrix functions $\tilde{S}_{n}^{*}\left(j_{0}\right)$ we will use the scaling properties of $S_{n}$ when the spins $x, j$ and $j_{0}$ are scaled. Namely, let us assume

\footnotetext{
${ }^{1}$ In the case of the loop spin network, a numerical investigation has given 11.000 configurations with small spins. The maximal number of small spins was 9 out of 22 spins.
} 
that $S_{n}$ satisfy

$$
S_{n}\left(\Lambda x, \Lambda j, \Lambda j_{0}\right)=\Lambda^{2}\left[R_{n}\left(x, j, j_{0}\right)+O(1 / \Lambda)\right]
$$

The scaling (3.5) will be consistent with the approximation (3.4) if the stationary points have the form

$$
x^{*}=\mu_{1}^{*} j_{0}+\mu_{0}^{*}+O\left(j_{0}^{-1}\right), \quad j^{*}=\nu_{1}^{*} j_{0}+\nu_{0}^{*}+O\left(j_{0}^{-1}\right) .
$$

The form (3.6) of the stationary points, together with the scaling (3.5) and the approximation (3.4) imply

$$
\tilde{S}^{*}=B^{*}+C^{*} j_{0}^{-1}+O\left(j_{0}^{-2}\right),
$$

where $B^{*}$ and $C^{*}$ are constant (independent of $j_{0}$ ) matrices. If $S_{n}$ are such that $\nu_{1}^{*}=1$, which will be imposed by the choice (2.3), then we will obtain the asymptotics of the same type as (1.4). However, in order to make a final comparison we need to calculate the matrices $B^{*}$ and $C^{*}$.

\section{$4 \quad \Psi_{+}$integral}

Let us now make a more detailed analysis of the $\Psi_{+}$integral. In order to calculate the matrices $A^{*}$ and $B^{*}$, it will be convenient to introduce the scaling parameter $\Lambda$ into the integral $\Psi_{+}$through the following change of variables:

$$
x+\frac{1}{2}=\Lambda y, \quad j+\frac{1}{2}=\Lambda y_{j}, \quad j_{0}+\frac{1}{2}=\Lambda y_{0} .
$$

Since $x=O(\Lambda)$ and $y=O(1)$, the integration domain $D_{+}$is then transformed into $D_{+}^{\prime}$, which is of $O(1) . \Lambda$ is essentially the same as $j_{0}$, since $y_{0}=O(1)$ and one can choose $y_{0}=1$. The Jacobian of the transformation is $\Lambda^{\omega}$, while the factors $2 a+1$ become $2 \Lambda y_{a}$. The spins $j$ and $j_{0}$ are changed into new variables $y_{j}$ i $y_{0}$, by using formula (4.1). The exponents $\mathrm{e}^{-\left(\lambda-j_{0}\right)^{2}}$ become $\mathrm{e}^{-\Lambda^{2}\left(y_{\lambda}-y_{0}\right)^{2}}$, so that we obtain

$$
\Psi_{+}\left(j, j_{0}\right) \approx 2^{A} \Lambda^{A+\omega} \int_{D_{+}^{\prime}} d^{\omega} y \prod_{a} y_{a} \prod_{\alpha} y_{\alpha} \prod_{\lambda} \frac{\mathrm{e}^{-\Lambda^{2}\left(y_{\lambda}-y_{0}\right)^{2}}}{y_{\lambda}} \prod_{v}\left\{6 j_{v}(\Lambda y)\right\} .
$$


Since every $6 j$ symbol in (4.2) can now be approximated by the PR formula (B.3), we obtain

$$
\begin{aligned}
\Psi_{+}\left(j, j_{0}\right) \approx & \frac{2^{A}}{(\sqrt{12 \pi})^{V}} \Lambda^{A+\omega-\frac{3 V}{2}} \\
& \times \int_{D_{+}^{\prime}} d^{\omega} y \prod_{a} y_{a} \prod_{\alpha} y_{\alpha} \prod_{\lambda} \frac{\mathrm{e}^{-\Lambda^{2}\left(y_{\lambda}-y_{0}\right)^{2}}}{y_{\lambda}} \prod_{v} \frac{\cos \left(\mathcal{S}_{v}(\Lambda, y)\right)}{\sqrt{V_{v}(y)}},
\end{aligned}
$$

where

$$
\mathcal{S}_{v}(\Lambda, y)=\Lambda \sum_{s \in v} y_{s} \theta_{s, v}(y)+\frac{\pi}{4}+\frac{1}{\Lambda} F_{D L}(y)+O\left(\frac{1}{\Lambda^{2}}\right) .
$$

The $O(\Lambda)$ term represents the Regge action, while the explicit form of the complicated $O(1 / \Lambda)$ term can be found in [10].

In order to apply Laplace's method, it is vital to rewrite the integrand as an exponential function, with the multiplicative factor $\Lambda$ in the exponent, and to determine the positions of the extremal points. The main problem is that the integrand is a product of cosine functions, which cannot be easily cast into an exponential form. This problem can be solved by using the approximation formula (C.4) derived in Appendix C:

$$
\cos x \approx \frac{1}{\vartheta_{4}\left(0, \mathrm{e}^{-\frac{\pi^{2}}{2}}\right)} \sum_{p \in \mathbb{Z}}(-1)^{p} \mathrm{e}^{-1 / 2(x-p \pi)^{2}} .
$$

This approximation has two main advantages. First, we avoid having to deal with complex-valued exponents, which would have been inevitable if we had employed the formula $\cos x=\left(\mathrm{e}^{\mathrm{i} x}+\mathrm{e}^{-\mathrm{i} x}\right) / 2$ and the corresponding stationary-phase method. Using the stationary-phase method would make the asymptotic analysis more complicated, because the corresponding stationary points will have the coordinates which are complex numbers. Second, we can "capture" the neighborhood of all extremal points at once, including those far away — when $p \sim O(\Lambda)$ - in a manifest manner. Specifically, every maximum and minimum at infinity can be labeled as $p=\Lambda m+n$, where $m, n \in \mathbb{Z}$ are of the order $O(1)$.

By applying (4.5) to (4.3), we have

$$
\cos \mathcal{S}_{v}(\Lambda, y)=\frac{1}{\vartheta_{4}\left(0, \mathrm{e}^{-\frac{\pi^{2}}{2}}\right)} \sum_{\substack{m_{v}, n_{v} \in \mathbb{Z} \\ m_{v}, n_{v} \ll \Lambda}}(-1)^{\Lambda m_{v}+n_{v}} \mathrm{e}^{-1 / 2\left(\mathcal{S}_{v}-\Lambda m_{v} \pi-n_{v} \pi\right)^{2}}
$$


Here it is crucial to note that the approximation is valid iff the exponent goes to zero, which will happen in the vicinity of extremal points $y_{0}^{*}$. We calculate the exact positions of these points by using the ansatz

$$
y_{N}^{*}=A_{N}+\frac{B_{N}}{\Lambda}+\frac{C_{N}}{\Lambda^{2}}+O\left(\frac{1}{\Lambda^{3}}\right) .
$$

Substituting this into the exponent and using (4.4), we obtain the necessary conditions ${ }^{2}$ for the extremal points $y_{0}^{*}$, in the form of two systems of equations for coefficients $A_{N}$ and $B_{N}$

$$
\begin{aligned}
& \sum_{s \in v} A_{s} \theta_{s, v}(A)=m_{v} \pi, \\
& \sum_{s \in v} B_{s} \theta_{s, v}(A)=n_{v} \pi-\frac{\pi}{4} .
\end{aligned}
$$

Of course, these equations are not a sufficient condition to determine the extremal points, simply because we are yet to discuss the full integrand in (4.3). The additional missing equations will be determined later.

Returning now to (4.6), we will use (4.4) to expand the exponent in (4.6) in powers of $1 / \Lambda$ in order to extract the leading $\Lambda^{2}$ term, as needed for the saddle-point method

$$
\begin{gathered}
-\Lambda^{2}\left[\frac{1}{2}\left(\sum_{s \in v} y_{s} \theta_{s, v}-m_{v} \pi\right)^{2}+\frac{1}{\Lambda}\left(\sum_{s \in v} y_{s} \theta_{s, v}-m_{v} \pi\right)\left(\frac{\pi}{4}-n_{v} \pi\right)\right. \\
\left.+\frac{1}{2 \Lambda^{2}}\left(\frac{\pi}{4}-n_{v} \pi\right)^{2}+\frac{1}{\Lambda^{2}}\left(\sum_{s \in v} y_{s} \theta_{s, v}-m_{v} \pi\right) F_{D L}+O\left(\frac{1}{\Lambda^{3}}\right)\right] .
\end{gathered}
$$

This can be done for all cosine functions in (4.3), so in the end we obtain

$$
\begin{aligned}
\Psi_{+}\left(j, j_{0}\right) \approx & \frac{2^{A} \Lambda^{A+\omega-\frac{3 V}{2}}}{\left[\sqrt{12 \pi} \vartheta_{4}\left(0, \mathrm{e}^{-\frac{\pi^{2}}{2}}\right)\right]^{V}} \sum_{\substack{m_{1}, n_{1} \in \mathbb{Z} \\
m_{1}, n_{1} \ll \Lambda}} \cdots \sum_{\substack{m_{V}, n_{V} \in \mathbb{Z} \\
m_{V}, n_{V} \ll \Lambda}}(-1)^{\sum_{v}\left(\Lambda m_{v}+n_{v}\right)} \\
& \times \int_{D_{+}^{\prime}} d^{\omega} y \mathrm{e}^{\Lambda^{2} S(\Lambda, y)} .
\end{aligned}
$$

\footnotetext{
${ }^{2}$ Here we can also note the following detail. In principle, we could have written the integer $p$ from equation (4.5) in the more general form, which includes some higher power of $\Lambda$, like $p=\Lambda^{2} l+\Lambda m+n$. In that case, we would obtain a consistency condition $l=0$ in addition to equations (4.8) and (4.9). Therefore, our choice $p=\Lambda m+n$ is actually the most general non-trivial one, dictated by the linear $\Lambda$-dependence in (4.4).
} 
This expression can be explicitly integrated via the Laplace method, term by term.

Here the phase has the general form

$$
S(\Lambda, y) \equiv S_{0}(y)+\frac{1}{\Lambda} S_{1}(y)+\frac{1}{\Lambda^{2}} S_{2}(y)+O\left(\frac{1}{\Lambda^{3}}\right),
$$

and we have explicitly

$$
\begin{aligned}
S_{0}(y)= & -C \sum_{\lambda}\left(y_{\lambda}-y_{0}\right)^{2}-\frac{1}{2} \sum_{v}\left(\sum_{s \in v} y_{s} \theta_{s, v}-m_{v} \pi\right)^{2}, \\
S_{1}(y)= & -\sum_{v}\left(\sum_{s \in v} y_{s} \theta_{s, v}-m_{v} \pi\right)\left(\frac{\pi}{4}-n_{v} \pi\right), \\
S_{2}(y)= & \sum_{a} \ln y_{a}-\frac{1}{2} \sum_{v}\left[\ln V_{v}+\left(\frac{\pi}{4}-n_{v} \pi\right)^{2}\right. \\
& \left.+2\left(\sum_{s \in v} y_{s} \theta_{s, v}-m_{v} \pi\right) F_{D L}\right] .
\end{aligned}
$$

The major gain here lies in the fact that there is a systematic expansion of the phase in powers of $1 / \Lambda$, while the leading term is of the order $O(1)$. As it will turn out, this will become very important as we go on to study the asymptotic behavior of the whole wavefunction. We shall systematically calculate everything up to terms of the order $O\left(1 / \Lambda^{3}\right)$, since this is the lowest self-consistent approximation for the Laplace method, as we shall see below. This is also the reason why we keep the $O(1 / \Lambda)$ term in (4.4).

\section{Stationary-point approximation}

We have written the wavefunction (4.10) in the form required for the application of the Laplace method. However, given that the integral is multidimensional, there are certain complications. The first step is to expand the phase into a power series around an extremal point $y_{N}^{*}$. We have to cast the series in the form which separates the variables $y_{j}$, which are not to be integrated over, from the internal $y_{N}(N \in \mathcal{I})$ variables

$$
S(\Lambda, y)=S\left(y_{n}^{*}, y_{j}^{*}\right)+\sum_{N \in \mathcal{I}} \frac{\partial S}{\partial y_{N}}\left(y_{N}-y_{N}^{*}\right)+\sum_{j} \frac{\partial S}{\partial y_{j}}\left(y_{j}-y_{j}^{*}\right)
$$




$$
\begin{aligned}
& +\frac{1}{2} \sum_{M, N \in \mathcal{I}} \frac{\partial^{2} S}{\partial y_{M} \partial y_{N}}\left(y_{M}-y_{M}^{*}\right)\left(y_{N}-y_{N}^{*}\right) \\
& +\sum_{\substack{j \in \mathcal{I} \\
j}} \frac{\partial^{2} S}{\partial y_{M} \partial y_{j}}\left(y_{M}-y_{M}^{*}\right)\left(y_{j}-y_{j}^{*}\right) \\
& +\frac{1}{2} \sum_{j, j^{\prime}} \frac{\partial^{2} S}{\partial y_{j} \partial y_{j^{\prime}}}\left(y_{j}-y_{j}^{*}\right)\left(y_{j^{\prime}}-y_{j^{\prime}}^{*}\right)+\cdots
\end{aligned}
$$

Given that all derivatives above are evaluated at an extremal point $y_{N}^{*}$, the terms with first derivatives vanish. Also, since all the differences $y_{N}-y_{N}^{*}$ go to zero as $1 / \Lambda$, due to equations (4.8) and (4.9), we can neglect the terms of the order $\left(y-y^{*}\right)^{3}$ and higher. This leaves us with

$$
\begin{aligned}
S(\Lambda, y)= & S^{*}+\frac{1}{2}\left(y-y^{*}\right)^{T} S^{\prime \prime}\left(y-y^{*}\right)+\left(y_{j}-y_{j}^{*}\right)^{T} \dot{S}^{\prime}\left(y-y^{*}\right) \\
& +\frac{1}{2}\left(y_{j}-y_{j}^{*}\right)^{T} \ddot{S}\left(y_{j}-y_{j}^{*}\right),
\end{aligned}
$$

where we have introduced a shorter matrix notation,

$$
\begin{gathered}
S^{*}=S\left(\Lambda, y^{*}\right), \quad S^{\prime \prime}=\left.\frac{\partial^{2} S}{\partial y_{M} \partial y_{N}}\right|_{y^{*}}, \\
\dot{S}^{\prime}=\left.\frac{\partial^{2} S}{\partial y_{j} \partial y_{M}}\right|_{y^{*}}, \quad \ddot{S}=\left.\frac{\partial^{2} S}{\partial y_{j} \partial y_{j^{\prime}}}\right|_{y^{*}}(M, N \in \mathcal{I}) .
\end{gathered}
$$

The matrices $S^{\prime \prime}, \dot{S}^{\prime}$ and $\ddot{S}$ are of the type $\omega \times \omega, J \times \omega$ and $J \times J$, respectively. At this point, we see that keeping all terms of the order up to $O\left(1 / \Lambda^{3}\right)$ is necessary, since if we had kept only terms up to $O\left(1 / \Lambda^{2}\right)$, the phase would have been approximated by a constant, and the Laplace method would have not worked.

Now the integrals in the wavefunction obtain the form

$$
\begin{aligned}
I= & \int_{D_{+}^{\prime}} d^{\omega} y \mathrm{e}^{\Lambda^{2} S(\Lambda, y)} \\
= & \mathrm{e}^{\Lambda^{2} S^{*}} \mathrm{e}^{\frac{\Lambda^{2}}{2}\left(y_{j}-y_{j}^{*}\right)^{T} \ddot{S}\left(y_{j}-y_{j}^{*}\right)} \\
& \times \int_{D_{+}^{\prime}} d^{\omega} y \mathrm{e}^{\frac{\Lambda^{2}}{2}\left(y-y^{*}\right)^{T} S^{\prime \prime}\left(y-y^{*}\right)+\Lambda^{2}\left(y_{j}-y_{j}^{*}\right)^{T} \dot{S}^{\prime}\left(y-y^{*}\right)} .
\end{aligned}
$$


At this step it is important to note that we have one integral of this type for every allowed value of $m_{v}$ and $n_{v}$, and for every extremal point $y_{N}^{*}$ which is inside the integration domain $D_{+}^{\prime}$. The Gaussian form of all the terms in (4.6) guarantees that all extremal points of the integrand are maxima, which in turn means that all eigenvalues of the matrix $S^{\prime \prime}$ are negative or zero.

This allows us to expand the integration domain $D_{+}^{\prime}$ to $\mathbb{R}^{\omega}$, since the eventual "exterior" extremal points are not taken into account while everything else is negligible in the limit $\Lambda \rightarrow \infty$. The zero eigenvalues contribute with linearly divergent terms, but this can be regularized in the sense of the generalized Gaussian integral (E.2) (see Appendix E). Even when the integration domain is extended to $\mathbb{R}^{\omega}$ the integral converges (or has a constant divergent contribution), and the integration can be explicitly performed by using the formula (E.2).

Therefore, after a suitable orthogonal change of variables $z=O\left(y-y^{*}\right)$ which brings $S^{\prime \prime}$ and $\dot{S}^{\prime}$ in a block-diagonal form, we perform the integration and obtain

$$
\begin{aligned}
I= & \sum_{y^{*} \in D_{+}^{\prime}} \mathrm{e}^{\Lambda^{2} S^{*}} \mathrm{e}^{\frac{\Lambda^{2}}{2}\left(y_{j}-y_{j}^{*}\right)^{T} \ddot{S}\left(y_{j}-y_{j}^{*}\right)}\left[\int_{\mathbb{R}} d z\right]^{\omega-r} \\
& \times \frac{1}{\Lambda^{r}} \sqrt{\frac{(2 \pi)^{r}}{|\operatorname{det} M|}} \mathrm{e}^{-\frac{\Lambda^{2}}{2}\left(y_{j}-y_{j}^{*}\right)^{T} N M^{-1} N^{T}\left(y_{j}-y_{j}^{*}\right)} .
\end{aligned}
$$

Here $r$ is the rank of matrix $S^{\prime \prime}$, while $M=M_{S^{\prime \prime}}$ (as defined in Appendix E). It is important to note that the orthogonal change of basis which brings $S^{\prime \prime}$ into a block-diagonal form does not necessarily guarantee that $\dot{S}^{\prime}$ will also reduce to zero in the null-space of $S^{\prime \prime}$. In other words, if $K$ denotes the null-space projector of $S^{\prime \prime}$, it is not guaranteed that $\dot{S}^{\prime} K=0$, which was assumed in the above equation. However, if this assumption is violated, we can immediately conclude that $\Psi_{+}$does not have the desired asymptotics, because of the integral of a linear exponential function. Nevertheless, numerical investigations (see Subsections 7.1 and 7.2) suggest that $\dot{S}^{\prime} K$ is indeed zero, so we proceed assuming that this is satisfied.

Using the fact that $\Lambda\left(y_{j}-y_{j}^{*}\right)=x_{j}-j^{*}$ we can switch back to the variables $j$ and $j_{0}$, and write the resulting integral as

$$
I=\sum_{y^{*} \in D_{+}^{\prime}} \frac{\mathrm{e}^{\Lambda^{2} S^{*}}}{\Lambda^{r}} \sqrt{\frac{(2 \pi)^{r}}{|\operatorname{det} M|}}\left[\int_{\mathbb{R}} d z\right]^{\omega-r} \mathrm{e}^{-\left(x-j^{*}\right)^{T} \tilde{S}\left(x-j^{*}\right)}
$$


where we have introduced the matrix

$$
\tilde{S} \equiv-\frac{1}{2}\left(\ddot{S}-N M^{-1} N^{T}\right) .
$$

This matrix is of type $J \times J$, and represents the key ingredient of the calculation. It is known as the Schur complement and is well-studied in the general matrix theory (see [11] and Appendix F).

Finally, we substitute this result back in equation (4.10), and obtain

$$
\begin{aligned}
& \Psi_{+}\left(j, j_{0}\right) \approx \frac{2^{A} \Lambda^{A+\omega-\frac{3 V}{2}}}{\left[\sqrt{12 \pi} \vartheta_{4}\left(0, \mathrm{e}^{-\frac{\pi^{2}}{2}}\right)\right]^{V}} \\
& \sum_{\substack{m_{1}, n_{1} \in \mathbb{Z} \\
m_{1}, n_{1} \ll \Lambda}} \cdots \sum_{\substack{m_{V}, n_{V} \in \mathbb{Z} \\
m_{V}, n_{V} \ll \Lambda}} \sum_{y^{*} \in D_{+}^{\prime}}(-1)^{\sum_{v}\left(\Lambda m_{v}+n_{v}\right)} \\
& \\
& \times \frac{\mathrm{e}^{\Lambda^{2} S^{*}}}{\Lambda^{r}} \sqrt{\frac{(2 \pi)^{r}}{|\operatorname{det} M|}}\left[\int_{\mathbb{R}} d z\right]^{\omega-r} \mathrm{e}^{-\left(j-j^{*}\right)^{T} \tilde{S}\left(j-j^{*}\right)} .
\end{aligned}
$$

The matrices $M, N$ and their rank $r$ typically depend on the initial choices of parameters $m_{v}, n_{v}$ and $y_{N}^{*} \in D_{+}^{\prime}$. Due to the nature of the Laplace method, we should keep in the sum only those parameters, which give minimum $r$, and among those only the ones, which give maximum $S^{*}$. The resulting sum of the remaining Gaussian functions represents the asymptotic behavior of $\Psi_{+}$.

\section{Asymptotic behavior of the matrix $\tilde{S}$}

Let us now return to (4.10),

$$
\begin{aligned}
\Psi_{+}\left(j, j_{0}\right) \approx & \frac{2^{A} \Lambda^{A+\omega-\frac{3 V}{2}}}{\left[\sqrt{12 \pi} \vartheta_{4}\left(0, \mathrm{e}^{-\frac{\pi^{2}}{2}}\right)\right]^{V}} \sum_{\substack{m_{1}, n_{1} \in \mathbb{Z} \\
m_{1}, n_{1} \ll \Lambda}} \cdots \sum_{\substack{m_{V}, n_{V} \in \mathbb{Z} \\
m_{V}, n_{V} \ll \Lambda}}(-1)^{\sum_{v}\left(\Lambda m_{v}+n_{v}\right)} \\
& \times \int_{D_{+}^{\prime}} d^{\omega} y \mathrm{e}^{\Lambda^{2} S(\Lambda, y)} .
\end{aligned}
$$

The phase $S(\Lambda, y)$ can be expanded into a power series around an extremal point $y_{N}^{*}$ (the first derivatives vanish, while the third and higher-order 
derivatives are of $\left.O\left(1 / \Lambda^{3}\right)\right)$, so that

$$
S(\Lambda, y)=S\left(\Lambda, y^{*}\right)+\frac{1}{2}\left(y-y^{*}\right)^{T} \Delta\left(y-y^{*}\right) .
$$

Here the $\Delta$ matrix is defined as

$$
\left.\Delta_{M N} \equiv \frac{\partial^{2} S(\Lambda, y)}{\partial y_{M} \partial y_{N}}\right|_{y=y^{*}} .
$$

It is of type $\Omega \times \Omega$, and it is convenient because it can be decomposed into blocks of size $J$ and $\omega$

$$
\Delta \equiv\left[\Delta_{M N}\right]=\left[\begin{array}{c|c}
\ddot{S} & \dot{S}^{\prime} \\
\hline\left(\dot{S}^{\prime}\right)^{T} & S^{\prime \prime}
\end{array}\right] .
$$

After an orthogonal transformation of the basis, the matrices $S^{\prime \prime}$ and $\dot{S}^{\prime}$ will reduce simultaneously into a block-diagonal form, so that the $\Delta$ matrix will obtain the following form:

$$
\Delta=\left[\begin{array}{c|cc}
\ddot{S} & N & 0 \\
\hline N^{T} & M & 0 \\
0 & 0 & 0
\end{array}\right] .
$$

Integration over the zeroes in (6.1) will boil down to a trivial divergent part, as seen in (5.2), and we can consider only the non-zero block. Note that $\tilde{S}$ matrix defined by (5.1) is actually (minus one half of) the Schur complement of the non-zero block of the $\Delta$ matrix.

At this point we apply the theorem from Appendix F, which states the following:

- $R=r+\rho$, where $R, r$ and $\rho$ are ranks of matrices $\Delta, S^{\prime \prime}$ and $\tilde{S}$ respectively;

- if $\rho=J$ then $\operatorname{det} M_{\Delta}= \pm \operatorname{det} M \operatorname{det} 2 \tilde{S}$;

- if $0<\rho<J$ then $\operatorname{det} M_{\Delta}\left(\operatorname{det} B_{4}\right)^{2}= \pm \operatorname{det} M \operatorname{det} M_{2 \tilde{S}}$;

where the signs depend on even/odd rank of $\tilde{S}$. See Appendix F for a proof of the theorem and the definition of the matrix $B_{4}$. 
The $\Delta$ matrix can be expanded into power series

$$
\Delta=\Delta_{0}+\frac{1}{\Lambda} \Delta_{1}+\frac{1}{\Lambda^{2}} \Delta_{2}+\cdots
$$

and as we shall see in the next section, the first non-zero leading term in the series is always $\Delta_{0}$. Consequently the determinant for the $\Delta$ matrix is given by

$$
\operatorname{det} \Delta=\operatorname{det} \Delta_{0}+O\left(\frac{1}{\Lambda}\right)
$$

Given that $M$ is a submatrix of $\Delta$, it follows that

$$
\operatorname{det} M=\operatorname{det} M_{0}+O\left(\frac{1}{\Lambda}\right)
$$

Assume now that

$$
\tilde{S}=\frac{1}{\Lambda^{n}} \tilde{S}_{n}+O\left(\frac{1}{\Lambda^{n+1}}\right)
$$

where $n \in \mathbb{N}_{0}$. If the rank $\rho$ of $\tilde{S}$ is positive, we have

$$
\operatorname{det}(2 \tilde{S})=\frac{1}{\Lambda^{n \rho}} \operatorname{det}\left(2 \tilde{S}_{n}\right)+O\left(\frac{1}{\Lambda^{n \rho+1}}\right)
$$

Equation (6.2) implies three distinct possibilities. If $\rho=J$, then we can use the first identity for determinants from the theorem, and obtain the equation

$$
\operatorname{det} \Delta_{0}= \pm \frac{1}{\Lambda^{n J}} \operatorname{det} M \operatorname{det}\left(2 \tilde{S}_{n}\right)+O\left(\frac{1}{\Lambda}\right)
$$

which is consistent if and only if $n=0$. If $0<\rho<J$, we can use the second identity for determinants, and obtain the equation:

$$
\operatorname{det} \Delta_{0}\left(\operatorname{det} B_{4}\right)^{2}= \pm \frac{1}{\Lambda^{n \rho}} \operatorname{det} M \operatorname{det}\left(2 \tilde{S}_{n}\right)+O\left(\frac{1}{\Lambda}\right),
$$

which is consistent if and only if $n=0$, due to the fact that $\operatorname{det} B_{4} \sim O(1)$ (see Remark F.3 in Appendix F). Finally, if $\rho=0$ we have $\tilde{S}=0$.

Therefore, we have essentially two possible situations. If $\rho=0$, then the matrix $\tilde{S}$ is equal to zero, because $\rho$ is its rank. This implies that the wavefunction (5.2) is constant in the leading order of $\Lambda$, i.e., the $j$ dependence only appears in the subleading terms, which are of the type $\left(j-j_{0}\right)^{3}$ and higher. On the other hand, if $n=0, \tilde{S}$ is different from zero in the leading order, so that (5.2) is a Gaussian, but not of the required type (1.4). Hence, the large-spin asymptotics of $\Psi_{+}$is never a Gaussian function of type (1.4). 


\section{Computation of the matrix $\tilde{S}$}

The main result of the previous section has been obtained under the assumption that the matrix $\Delta$ has a leading contribution of $O(1)$ in $\Lambda$. In this section, we will demonstrate this by an explicit computation for some concrete spin-network diagrams. In order to do so, we need to explicitly find an extremal point $y_{N}^{*}$ and evaluate the corresponding $\Delta$ for a given spin network.

An extremal point is defined by

$$
\frac{\partial S}{\partial y_{N}}=0, \quad \text { where } y^{*} \in \Delta^{\prime}, \quad N \in \mathcal{N},
$$

where we must also take into account the consistency conditions (4.8) and (4.9).

Differentiating (4.11) and (4.12), we obtain

$$
\begin{gathered}
\frac{\partial S_{0}}{\partial y_{N}}+\frac{1}{\Lambda} \frac{\partial S_{1}}{\partial y_{N}}+\frac{1}{\Lambda^{2}} \frac{\partial S_{2}}{\partial y_{N}}=O\left(\frac{1}{\Lambda^{3}}\right) \\
\frac{\partial S_{0}}{\partial y_{N}}=-2 C\left(y_{N}-y_{0}\right) \delta_{N, \lambda}-\sum_{\substack{v \\
N \in v}} \theta_{N, v}\left(\sum_{s \in v} y_{s} \theta_{s, v}-m_{v} \pi\right) \\
\frac{\partial S_{1}}{\partial y_{N}}=-\sum_{v \in v} \theta_{N, v}\left(\frac{\pi}{4}-n_{v} \pi\right) \\
\frac{\partial S_{2}}{\partial y_{N}}=\frac{1}{y_{N}} \delta_{N, a}-\sum_{N \in v}\left[\frac{1}{2 V_{v}} \frac{\partial V_{v}}{\partial y_{N}}+\theta_{N, v} F_{D L}+\left(\sum_{s \in v} y_{s} \theta_{s, v}-m_{v} \pi\right) \frac{\partial F_{D L}}{\partial y_{N}}\right],
\end{gathered}
$$

where we have used the Schläfli differential identity (B.2) for a tetrahedron

$$
\sum_{s \in v} y_{s} \frac{\partial \theta_{s, v}}{\partial y_{N}}=0, \quad \forall N, v
$$

We will also need second derivatives:

$$
\frac{\partial^{2} S_{0}}{\partial y_{M} \partial y_{N}}=-2 C \delta_{M N} \delta_{N, \lambda}-\sum_{M, N \in v}\left[\theta_{M, v} \theta_{N, v}+\frac{\partial \theta_{N, v}}{\partial y_{M}}\left(\sum_{s \in v} y_{s} \theta_{s, v}-m_{v} \pi\right)\right],
$$




$$
\begin{aligned}
\frac{\partial^{2} S_{1}}{\partial y_{M} \partial y_{N}}= & -\sum_{M, N \in v} \frac{\partial \theta_{N, v}}{\partial y_{M}}\left(\frac{\pi}{4}-n_{v} \pi\right) \\
\frac{\partial^{2} S_{2}}{\partial y_{M} \partial y_{N}}= & -\frac{1}{y_{N}^{2}} \delta_{M N} \delta_{N, a}-\sum_{\substack{v \\
N \in v}}\left[\frac{1}{2 V_{v}} \frac{\partial^{2} V_{v}}{\partial y_{M} \partial y_{N}}-\frac{1}{2 V_{v}^{2}} \frac{\partial V_{v}}{\partial y_{M}} \frac{\partial V_{v}}{\partial y_{N}}+\frac{\partial \theta_{N, v}}{\partial y_{M}} F_{D L}\right. \\
& \left.+\theta_{N, v} \times \frac{\partial F_{D L}}{\partial y_{M}}+\theta_{M, v} \frac{\partial F_{D L}}{\partial y_{N}}+\left(\sum_{s \in v} y_{s} \theta_{s, v}-m_{v} \pi\right) \frac{\partial^{2} F_{D L}}{\partial y_{M} \partial y_{N}}\right]
\end{aligned}
$$

A solution of the system (7.2) can be obtained perturbatively in $1 / \Lambda$ via the ansatz (4.7),

$$
y_{N}^{*}=A_{N}+\frac{B_{N}}{\Lambda}+\frac{C_{N}}{\Lambda^{2}}+O\left(\frac{1}{\Lambda^{3}}\right) .
$$

In the lowest order, we obtain a non-linear system for $A_{N}$,

$$
2 C\left(A_{N}-y_{0}\right) \delta_{N, \lambda}+\sum_{v \in v} \theta_{N, v}(A)\left(\sum_{s \in v} A_{s} \theta_{s, v}(A)-m_{v} \pi\right)=0
$$

which can be reduced to a simple equation using (4.8),

$$
A_{\lambda}=y_{0}
$$

This was already guessed before based on the analysis that the extremal point must be in the vicinity of extremal points of the cosine and the Gaussian functions $\mu\left(x_{\lambda}\right)$.

At $O(1 / \Lambda)$ order we obtain also a linear system of equations for the $B_{N}$ coefficients

$$
\begin{aligned}
\sum_{M} & {\left[2 C \delta_{M N} \delta_{N, \lambda}+\left.\sum_{\substack{v \\
M, N \in v}} \frac{\partial \theta_{N, v}}{\partial y_{M}}\right|_{A}\left(\sum_{s \in v} A_{s} \theta_{s, v}(A)-m_{v} \pi\right)\right.} \\
& \left.+\sum_{\substack{v \\
M, N \in v}} \theta_{N, v}(A) \theta_{M, v}(A)\right] B_{M}=-\sum_{\substack{v \\
N \in v}} \theta_{N, v}(A)\left(\frac{\pi}{4}-n_{v} \pi\right),
\end{aligned}
$$

which can be simplified using (4.8) and (4.9) to

$$
B_{\lambda}=0 \text {. }
$$


Similarly, by applying (4.8) and (4.9) at the $O\left(1 / \Lambda^{2}\right)$ order, we obtain a linear system for the coefficients $C_{N}$

$$
\begin{aligned}
\sum_{M} & {\left[2 C \delta_{M N} \delta_{N, \lambda}+\sum_{\substack{v, N \in v \\
v}} \theta_{N, v}(A) \theta_{M, v}(A)\right] C_{M} } \\
= & -\frac{1}{A_{N}} \delta_{N, a}-\sum_{\substack{v \\
N \in v}}\left(\left.\frac{1}{2 V_{v}} \frac{\partial V_{v}}{\partial y_{N}}\right|_{A}+\theta_{N, v}(A) F_{D L}(A)\right) .
\end{aligned}
$$

Let us now write the complete set of equations, which determine the extremal point $y^{*}$

- equations for $A_{N}$ :

$$
\sum_{s \in v} A_{s} \theta_{s, v}(A)=m_{v} \pi, \quad A_{\lambda}=y_{0},
$$

- equations for $B_{N}$ :

$$
\sum_{s \in v} B_{s} \theta_{s, v}(A)=n_{v} \pi-\frac{\pi}{4}, \quad B_{\lambda}=0,
$$

- equations for $C_{N}$ :

$$
\begin{aligned}
\sum_{M} & {\left[2 C \delta_{M N} \delta_{N, \lambda}+\sum_{\substack{v \\
M \in v}} \theta_{N, v}(A) \theta_{M, v}(A)\right] C_{M} } \\
= & -\frac{1}{A_{N}} \delta_{N, a}-\sum_{\substack{v \\
N \in v}}\left(\left.\frac{1}{2 V_{v}} \frac{\partial V_{v}}{\partial y_{N}}\right|_{A}+\theta_{N, v}(A) F_{D L}(A)\right)
\end{aligned}
$$

Before we engage in finding solutions to these equations, let us introduce some notation and discuss the form of the second derivatives of the phase $S(\Lambda, y)$. We will introduce the following shorter notation for various derivatives evaluated at the particular extremal point:

$$
\begin{aligned}
& \theta_{N, v}=\theta_{N, v}(A), \quad \theta_{M N, v}=\left.\frac{\partial \theta_{N, v}}{\partial y_{M}}\right|_{A}, \quad \theta_{M N s, v}=\left.\frac{\partial^{2} \theta_{N, v}}{\partial y_{s} \partial y_{M}}\right|_{A}, \\
& \theta_{M N p q, v}=\left.\frac{\partial^{3} \theta_{N, v}}{\partial y_{p} \partial y_{q} \partial y_{M}}\right|_{A}, \\
& V_{v}=V_{v}(A), \quad V_{N, v}=\left.\frac{\partial V_{v}}{\partial y_{N}}\right|_{A}, \quad V_{M N, v}=\left.\frac{\partial^{2} V_{v}}{\partial y_{M} \partial y_{N}}\right|_{A} .
\end{aligned}
$$


By using (7.3) and the ansatz (4.7) for $y^{*}$, as well as equations (4.8) and (4.9), we obtain

$$
\begin{aligned}
\Delta_{M N}= & -\left\{2 C \delta_{M N} \delta_{N, \lambda}+\sum_{M, N \in v} \theta_{M, v} \theta_{N, v}\right\} \\
& -\frac{1}{\Lambda}\left\{\sum_{\substack{v \\
M, N \in v}} B_{s \in v}\left(\theta_{M, v} \theta_{N s, v}+\theta_{N, v} \theta_{M s, v}\right)\right\} \\
& -\frac{1}{\Lambda^{2}}\left\{\frac{1}{A_{N}^{2}} \delta_{M N} \delta_{N, a}+\sum_{\substack{v \\
M, N \in v}}\left[\frac{V_{M N, v}}{2 V_{v}}-\frac{V_{M, v} V_{N, v}}{2 V_{v}^{2}}+\theta_{M N, v} F_{D L}\right.\right. \\
& \left.+\left.\theta_{N, v} \frac{\partial F_{D L}}{\partial y_{M}}\right|_{A}+\left.\theta_{M, v} \frac{\partial F_{D L}}{\partial y_{N}}\right|_{A}\right] \\
& +\sum_{, v}\left[\theta_{M, v} \sum_{s \in v} C_{s} \theta_{N s, v}+\theta_{N, v} \sum_{s \in v} C_{s} \theta_{M s, v}\right. \\
& \left.+\frac{1}{2} \theta_{M, v} \sum_{p, q \in v} B_{p} B_{q} \theta_{N p q, v}+\frac{1}{2} \theta_{N, v} \sum_{p, q \in v} B_{p} B_{q} \theta_{M p q, v}\right] \\
& +\sum_{v}^{v} \theta_{M N, v}\left[\sum_{s \in v} C_{s} \theta_{s, v}+\left(\frac{\pi}{4}-n_{v} \pi\right) \sum_{s \in v} B_{s} \theta_{M N s, v}\right. \\
& M, N \in v \\
& \left.\left.+\frac{1}{2} \sum_{p, q \in v} B_{p} B_{q} \theta_{p q, v}\right]\right\}+O\left(\frac{1}{\Lambda^{3}}\right) .
\end{aligned}
$$

Therefore, after finding an explicit extremal point, we have substituted the coefficients $A_{N}, B_{N}$ and $C_{N}$ into the above equations and obtained an explicit expression for the $\Delta$ matrix. The curly braces group all terms of orders $O(1), O(1 / \Lambda)$ and $O\left(1 / \Lambda^{2}\right)$, respectively. This demonstrates that the leading order of the $\Delta$ matrix is an $O(1)$ term.

Given that all of the above equations are fairly complicated, we will investigate them order by order in $\Lambda$. 


\subsection{The $O(1)$ approximation}

Let us rewrite equation (7.7) in this approximation as

$$
\sum_{s \in v} A_{s} \theta_{s, v}(A)=m_{v} \pi, \quad A_{\lambda}=y_{0} .
$$

We will also rewrite the expression for the $\Delta$ matrix as

$$
\Delta_{M N}=-2 C \delta_{M N} \delta_{N, \lambda}-\sum_{\substack{v \\ M \in v}} \theta_{M, v}(A) \theta_{N, v}(A) .
$$

The calculation of the matrix $\tilde{S}$ can be organized in the following way:

- calculate all extremal points $y_{N}^{*}=A_{N}$ by solving equation (7.11);

- for every extremal point obtained, calculate $\Delta$ matrix according to (7.12);

- split the $\Delta$ matrix to blocks $\ddot{S}, \dot{S}^{\prime}$ and $S^{\prime \prime}$;

- determine the rank of $S^{\prime \prime}$, and the null-space projector $K$ if the rank is less than $\omega$;

- check whether or not $\dot{S}^{\prime} K=0$; if it is non-zero the procedure fails and the wavefunction does not have Gaussian form;

- determine an orthogonal matrix $O$ which diagonalizes $K$ and use it to change to a basis where matrices $S^{\prime \prime}$ and $\dot{S}^{\prime}$ are block-diagonal; read-off the non-zero blocks $M$ and $N$;

- compute the matrix $\tilde{S}$ according to the equation

$$
\tilde{S} \equiv-\frac{1}{2}\left(\ddot{S}-N M^{-1} N^{T}\right) .
$$

The system of equations (7.11) is highly non-linear, and therefore extremely hard to solve. Nevertheless, one exact solution can be guessed

$$
\begin{gathered}
y_{N}^{*}=A_{N}=y_{0}, \quad \theta_{N, v}=\theta_{0} \equiv \pi-\arcsin \frac{2 \sqrt{2}}{3} \equiv \arccos \left(-\frac{1}{3}\right), \\
m_{v}=\frac{6 \theta_{0}}{\pi} y_{0} .
\end{gathered}
$$

We will refer to this solution as the "diagonal" solution. It corresponds to a situation where all the tetrahedra are equilateral, which is a very symmetrical configuration. This greatly simplifies the equations, and the required matrices can be calculated without additional approximations. In the case 
of the loop spin network and the theta spin network a computer calculation gives

$$
\dot{S}^{\prime} K=0, \quad R=r, \quad \tilde{S}=0 .
$$

This result agrees with the $\rho=0$ case discussed in Section 6 .

The calculation of the $\tilde{S}$ matrix can be also performed numerically for non-diagonal solutions. In fact, in the numerical approach, the hardest first step, solving the equation (7.11), can be completely sidestepped. This is due to the fact that $\Delta$ depends on $A_{N}$ only through the angles $\theta_{N, v}(A)$. Thus one can design an algorithm, which chooses the angles completely randomly from their domain $[0, \pi]$, which in principle also covers the angles obtained using any specific solution of (7.11). The constant $C>0$ can also be chosen randomly.

One such algorithm has been implemented on a computer to explicitly calculate the $\Delta$ matrix, the projector $K$, the rank of $S^{\prime \prime}$, then $\dot{S}^{\prime} K, M$ and $N$ and finally $\tilde{S}$. It has been executed 50 times with random initial data for the cases of a loop spin network and a theta spin network, and each execution gave the same result (7.13). The numerical precision of the calculation was $10^{-6}$. The precision can be arbitrarily increased at the expense of the execution time, and in 5 executions the precision was raised to $10^{-15}$, with no change in the result.

\subsection{The $O(1 / \Lambda)$ approximation}

Let us rewrite the all necessary equations up to $O\left(1 / \Lambda^{2}\right)$. The extremal points can be found using the ansatz

$$
y_{N}^{*}=A_{N}+\frac{B_{N}}{\Lambda}
$$

The coefficients $A_{N}$ and $B_{N}$ will be determined by the following equations:

$$
\begin{aligned}
& \sum_{s \in v} A_{s} \theta_{s, v}(A)=m_{v} \pi, \quad A_{\lambda}=y_{0}, \\
& \sum_{s \in v} B_{s} \theta_{s, v}(A)=n_{v} \pi-\frac{\pi}{4}, \quad B_{\lambda}=0 .
\end{aligned}
$$


$\Delta$ matrix is given as:

$$
\begin{aligned}
\Delta_{M N}= & -2 C \delta_{M N} \delta_{N, \lambda}-\sum_{M, N \in v}^{v} \theta_{M, v} \theta_{N, v} \\
& -\frac{1}{\Lambda}\left\{\sum_{\substack{v \\
M, N \in v}} \sum_{s \in v} B_{s}\left(\theta_{M, v} \theta_{N s, v}+\theta_{N, v} \theta_{M s, v}\right)\right\},
\end{aligned}
$$

where

$$
\theta_{N, v}=\theta_{N, v}(A), \quad \theta_{N s, v}=\left.\frac{\partial \theta_{N, v}}{\partial y_{s}}\right|_{A} .
$$

The procedure for computation of $\tilde{S}$ matrix is the same as in the $O(1)$ approximation, up to two additional steps. These two steps consist of solving the linear system of equations (7.15) for $B_{N}$ coefficients, and then expanding the resulting matrix into a power series in $1 / \Lambda$,

$$
\tilde{S}=\tilde{S}_{0}+\frac{\tilde{S}_{1}}{\Lambda}+O\left(\frac{1}{\Lambda^{2}}\right) .
$$

This case can be also analyzed analytically (by using the diagonal solution), as well as numerically (by randomly generating the $A_{N}$ coefficients). In contrast to the previous case, one should also always specify the $n_{v}$ parameters as a part of the initial data, in order to solve (7.15). One algorithm for this has also been implemented, and we obtained the same result (7.13) for a loop and a theta spin network.

\section{$8 \quad \Psi_{-}$integral}

The asymptotic analysis of $\Psi_{-}$can be done by using the same method as in the $\Psi_{+}$case. However, it turns out that $\Psi_{-}$analysis is considerably simpler due to qualitatively different nature of the scaling laws for the asymptotic expressions for $6 j$ symbols when some of the spins are not large.

The $\Psi_{-}$part of the wavefunction can be approximated by an analogous expression to $(4.2)$

$$
\begin{aligned}
\Psi_{-}\left(j, j_{0}\right) \approx & 2^{A} \Lambda^{A+\omega} \sum_{z} F(z) \int_{D_{-}^{\prime}} d^{\omega} y \\
& \times \prod_{a} y_{a} \prod_{\alpha} y_{\alpha} \prod_{\lambda} \frac{\mathrm{e}^{-\Lambda^{2}\left(y_{\lambda}-y_{0}\right)^{2}}}{y_{\lambda}} \prod_{v}\left\{6 j_{v}(\Lambda y, z)\right\},
\end{aligned}
$$


where the vector $z$ denotes the small spins, while the vector $\Lambda y$ denotes the large ones. We will sum over the small spins rather than integrate and the number $\omega$ of large spins is smaller than in the $\Psi_{+}$case, whereas $F(z)$ represents the part of the wavefunction, which does not depend on large spins $\Lambda y$. Which spins can remain small and which must be large depends on the triangle inequalities built into the $6 j$ symbols. These restrictions on the spins $z$ and $\Lambda y$ will also depend on the detailed topology of the spin network. However, these details will not affect the asymptotic analysis.

The next step is to use the asymptotic formulas for the $6 j$ symbols, but now we cannot use only the PR formula (B.3) since the $6 j$ symbols where not all of the spins are large will appear. The asymptotics of $6 j$ symbols with 3, 4 or 5 large spins is given in the appendix; see formulas (B.5), (B.6) and (B.7).

Let the asymptotic behavior of each $6 j$ symbol be described by a function $\phi_{i}(\Lambda y, z)$, so that

$$
\{6 j(\Lambda y, z)\} \approx \phi_{i}(\Lambda y, z) \text { as } \Lambda \rightarrow \infty
$$

where $i=3,4,5,6$ denotes the number of large spins. When $i<6$, we will refer to the corresponding $6 j$ symbols as degenerate. When $i=6$, we will refer to the corresponding $6 j$ symbol as a non-degenerate.

Note that in a degenerate case

$$
\phi_{i}(\Lambda y, z) \approx \Lambda^{\rho_{i}} f_{i}(y, z) \text { as } \Lambda \rightarrow \infty
$$

while in the non-degenarte case

$$
\phi(\Lambda y) \approx \Lambda^{\rho} \cos (\Lambda f(y)) \text { as } \Lambda \rightarrow \infty
$$

Let us write the integrand of $\Psi_{-}$integral in an exponential form suitable for stationary point approximation. The corresponding $\Delta$ matrix is given as before

$$
\Delta_{M N}^{-}=\left.\frac{\partial^{2} S_{-}(\Lambda y, z)}{\partial y_{M} \partial y_{N}}\right|_{y=y^{*}}
$$

where the new phase $S_{-}$is now proportional to $\sum_{v} \ln \phi_{v}(\Lambda y, z)$. It is easy to see that the $\Delta^{-}$matrix will contain the terms of the form

$$
\left(\frac{1}{\phi_{v}} \frac{\partial \phi_{v}}{\partial \Lambda y}\right)^{2}
$$


and

$$
\frac{1}{\phi_{v}} \frac{\partial^{2} \phi_{v}}{\partial \Lambda y \partial \Lambda y}
$$

All these terms are of $O\left(1 / \Lambda^{2}\right)$, since the asymptotic functions $\phi_{v}$ behave well when differentiated by $\Lambda y$, see (8.2). Furthermore, given that the $\Delta$ matrix should be evaluated at an extremal point $y^{*}$, one can see that the $6 j$ symbols which contain only three or four large spins give a sub-leading contribution, since they decrease monotonically in the limit $\Lambda \rightarrow \infty$ and thus do not have any extremal points. The $6 j$ symbols with 5 large spins have extremal points, but they also provide only a contribution of $O\left(1 / \Lambda^{2}\right)$ to the $\tilde{S}_{-}$matrix. The $O(1)$ contribution to $\tilde{S}_{-}$comes only from those $6 j$ symbols where all spins are large, due to the fact that the argument of the cosine function in (8.3) is proportional to $\Lambda$.

Therefore, the $\Psi_{-}$integral will have the same asymptotics as the $\Psi_{+}$ integral if there is at least one non-degenerate $6 j$ symbol contributing to $\tilde{S}_{-}$. If only the degenerate $6 j$ symbols are present, the contribution to $\tilde{S}_{-}$ will be of $O\left(1 / \Lambda^{2}\right)$.

\section{Conclusions}

We have shown that the leading term in the large-spin asymptotics of a flat-space wavefunction $\Psi_{k}\left(\Gamma, j_{0}\right) \equiv \Psi\left(j, j_{0}\right)$ is given by

$$
\begin{aligned}
\Psi\left(j, j_{0}\right) \approx & \sum_{n, p} N_{n p}^{-}\left(j_{0}\right) \mathrm{e}^{-\frac{1}{2}\left(j-j_{0}\right)^{T}\left(B_{n p}^{-}+\frac{1}{j_{0}^{2}} D_{n p}^{-}\right)\left(j-j_{0}\right)} \\
& +\sum_{n, q} N_{n q}^{+}\left(j_{0}\right) \mathrm{e}^{-\frac{1}{2}\left(j-j_{0}\right)^{T} B_{n q}^{+}\left(j-j_{0}\right)}
\end{aligned}
$$

where $n \in \mathbf{Z}, p \in S_{-}, q \in S_{+}$and $B$ and $D$ are constant (independent of $\left.j_{0}\right)$ matrices. The index sets $S_{ \pm}$correspond to the stationary points of the large-spin approximations $f_{ \pm}$of $f$ in the regions $D_{ \pm}$and the corresponding functions $N^{ \pm}\left(j_{0}\right)$ will be powers $j_{0}^{r_{ \pm}}, r_{ \pm} \in \mathbf{Q}$. In the case of a loop and a theta spin network, the computer results are consistent with $B_{n q}^{+}=C_{n q}^{+}=0$ for all $n$ and all $q$ which means that in those cases the matrix $\tilde{S}$ vanishes.

The asymptotics (9.1) is not of the type (1.4) required for the correct semi-classical limit. This means that the wavefunction $\Psi_{k}\left(\Gamma, j_{0}\right)$ will not give the correct graviton propagator asymptotics. One can argue that some other wavefunction may give the correct asymptotics, but the problem is 
to see what other wavefunction can replace $\Psi_{k}\left(\Gamma, j_{0}\right)$. Although our result applies only to the Euclidean LQG, one wonders what is the relevance of this result for the Lorentzian LQG, given that there is a strong belief that the Euclidean and the Lorentzian theories should be related by some kind of an analytic continuation. Note that Lorentzian analogs of the Euclidean wavefunctions used in this paper are not known. However, one can try use one of the recently proposed Lorentzian spin foam models [12-14] in order to construct a Lorentzian spin-network wavefunction. The large-spin asymptotics could be then computed by using essentially the same techniques as the ones introduced in this paper.

As far as the our result is concerned, there are certain caveats. The first caveat is that the obtained asymptotics is for the Ponzano-Regge regularization $\Psi_{k}^{P R}\left(\Gamma, j_{0}\right)$ of the zero-cosmological constant spin-network wavefunction $\Psi\left(\Gamma, j_{0}\right)$. The wavefunction $\Psi_{k}^{P R}\left(\Gamma, j_{0}\right)$ is different from the quantum group regularization $\Psi_{k}\left(\Gamma, j_{0}\right)$ of $\Psi\left(\Gamma, j_{0}\right)$, but the physics intuition suggests that the asymptotic behavior of $\Psi_{k}$ and $\Psi_{k}^{P R}$ for large $k$ should be essentially the same, up to constant factors; see for example [15]. However, it still remains to be proven that these two wavefunctions have the same semiclassical asymptotics.

The second caveat is that the construction of $\Psi_{k}$ wavefunctions is triangulation dependent and our calculations have been done for the simplest triangulation of $S^{3}$. However, it is not difficult to see that by taking a more complex triangulation, the corresponding asymptotics will not change qualitatively, because the corresponding $f\left(x, j, j_{0}\right)$ will be always a product of $6 j$ symbols and our method of computing the asymptotics is independent of the number of $6 j$ symbols. Similarly, one can hope that a special choice of the function $\mu(\lambda)$ can lead to a desired asymptotics. However, a generic $\mu(\lambda)$ gives a contribution to $\tilde{S}$ of $O(1)$ in $j_{0}$. Note that it is possible to fine-tune $\mu$ for a given spin network such that the $O(1)$ contributions cancel and the $O\left(1 / j_{0}\right)$ contributions are non-zero. However, this fine-tuning depends on the spin network and hence one cannot find a $\mu$, which will work for all spin networks.

\section{Acknowledgments}

We would like to thank John W. Barrett for discussions. AM was partially supported by the FCT grants PTDC/MAT/69635/2006 and PTDC/MAT/ 099880/2008. MV was supported by the FCT grants SFRH/BPD/46376/ 2008 and PTDC/MAT/099880/2008. 


\section{Appendix A The spin-network diagram}

The spin-network whose evaluation appears in (2.5), is given in the case of a single loop spin network with spin $j$ by the following diagram:

All spins take values from the set $\left\{0, \frac{1}{2}, 1, \frac{3}{2}, 2 \ldots, \frac{k}{2}\right\}$. Here $n$ is the degree of the corresponding quantum group $S U(2)_{q}$ such that $q^{2 k+2}=1$, i.e., $q=$ $\mathrm{e}^{\mathrm{i} \pi / k+2}$.

The evaluation of the above diagram, to which we refer as the amplitude, can be calculated via the following rules:

- The amplitude of the whole diagram is the product of the amplitudes for the vertices.

- The amplitude of a 3 -vertex is proportional to the amplitude of the corresponding tetrahedron spin network, as follows:
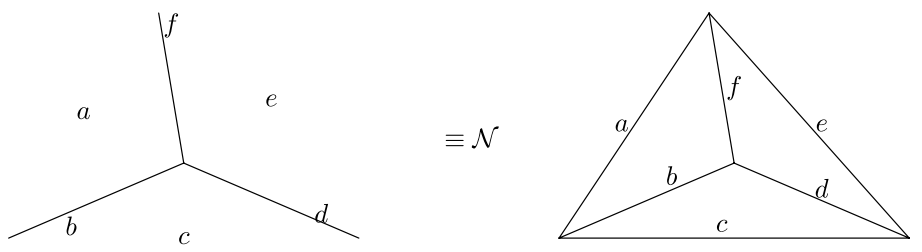

- The amplitude for a 4-vertex is proportional to the amplitude of the corresponding tetrahedron spin network, which is given by
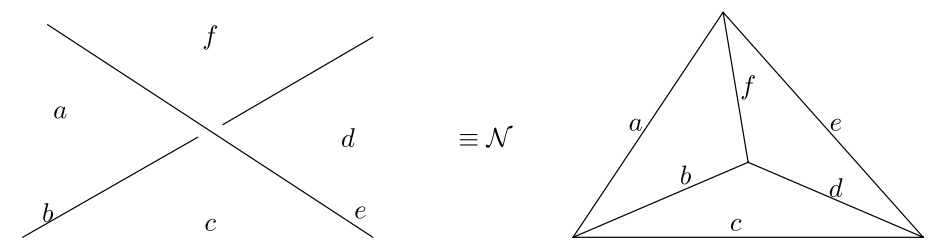

The normalization $\mathcal{N}$ is given by, see [4]

$$
\mathcal{N}=\frac{1}{\sqrt{|\Theta(a, b, c) \Theta(c, d, e) \Theta(a, e, f) \Theta(b, d, f)|}}
$$

where $\Theta(a, b, c)$ is the evaluation of the $\theta$-graph

$$
\Theta(a, b, c) \equiv(-1)^{a+b+c} \frac{[a+b-c]_{q} ![a+c-b]_{q} ![b+c-a]_{q} ![a+b+c+1]_{q} !}{[2 a]_{q} ![2 b]_{q} ![2 c]_{q} !}
$$




\section{Appendix B Tetrahedron spin network and the $6 j$ symbol}

The amplitude for a tetrahedron spin network is, roughly speaking, the value of the corresponding $6 j$ symbol. Following the conventions of [3] and [16], the amplitude is given by the equation

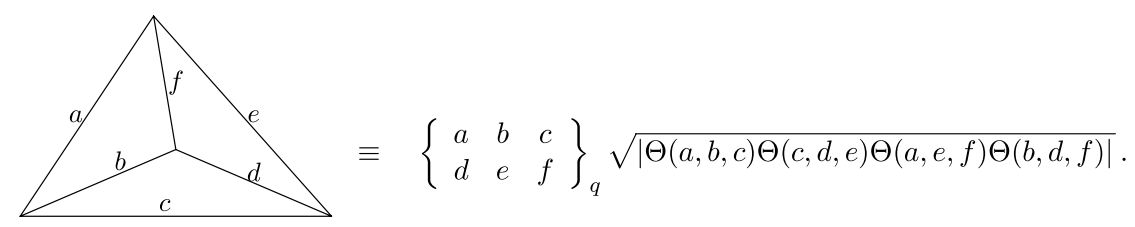

The tetrahedron associated to a $6 j$ symbol has the following geometric properties. The length of an edge colored by a spin $j$, is given by

$$
l=j+\frac{1}{2}
$$

The area of a face, with edge lengths $l_{1}, l_{2}, l_{3}$, is given by the Heron formula

$$
A_{123}=\sqrt{s\left(s-l_{1}\right)\left(s-l_{2}\right)\left(s-l_{3}\right)}, \quad \text { where } s \equiv \frac{1}{2}\left(l_{1}+l_{2}+l_{3}\right) .
$$

The volume of the tetrahedron is given by the Tartaglia determinant

$$
V^{2}=\frac{1}{288} \operatorname{det}\left[\begin{array}{ccccc}
0 & l_{12}^{2} & l_{13}^{2} & l_{14}^{2} & 1 \\
l_{12}^{2} & 0 & l_{23}^{2} & l_{24}^{2} & 1 \\
l_{13}^{2} & l_{23}^{2} & 0 & l_{34}^{2} & 1 \\
l_{14}^{2} & l_{24}^{2} & l_{34}^{2} & 0 & 1 \\
1 & 1 & 1 & 1 & 0
\end{array}\right]
$$

where $l_{i j}$ is the edge length between the vertices $i$ and $j$. Note that if we fix all six edge lengths, we could create in total 6 ! different tetrahedra. However, some of them are equivalent up to rotations and reflections. When this is taken into account (4! permutations of the 4 vertices of a tetrahedron), we end up with the total of $6 ! / 4 !=30$ possible inequivalent tetrahedra. Those 30 tetrahedra have different volumes, which means that if we provide six numbers $l_{i j}$, there is 30 inequivalent different ways to position them in the determinant above. 
A dihedral angle of a tetrahedron is given by the formula

$$
\sin \theta_{a}=\frac{3}{2} \frac{a V_{a b c d e f}}{A_{a b f} A_{a c e}} .
$$

The angle $\theta_{a}$ corresponds to the edge $a$, and it is constructed between the outward-normal vectors of the faces $a, b, f$ and $a, c, e$, such that the edge $d$ does not intersect the edge $a$. This angle is equal to $\pi-\varphi_{a}$, where $\varphi_{a}$ is the angle between the faces $a, b, f$ and $a, c, e$.

Regarding the above equation, once we fix the value of the right-hand side, there are in general two different angles, which satisfy the equation. This is a consequence of double-valuedness of the arcsine function on the $[0, \pi]$ codomain. However, when the edge lengths are given, $\theta_{a}$ can be expressed as

$$
\theta_{a}= \begin{cases}\pi-\arcsin \left(\frac{3}{2} \frac{a V_{a b c d e f}}{A_{a b f} A_{a c e}}\right), & \text { if } d \leqslant \frac{\sqrt{a^{2}\left(b^{2}+c^{2}+e^{2}+f^{2}-a^{2}\right)+\left(e^{2}-c^{2}\right)\left(b^{2}-f^{2}\right)}}{\sqrt{2} a}, \\ \arcsin \left(\frac{3}{2} \frac{a V_{a b c d e f}}{A_{a b f} A_{a c e}}\right), & \text { if } d>\frac{\sqrt{a^{2}\left(b^{2}+c^{2}+e^{2}+f^{2}-a^{2}\right)+\left(e^{2}-c^{2}\right)\left(b^{2}-f^{2}\right)}}{\sqrt{2} a} .\end{cases}
$$

Finally, it is convenient to note that the angle $\theta_{a}$ is unchanged if we scale all edge lengths of the tetrahedron, i.e., if we multiply them all with the same positive constant. This is easily visualized since the tetrahedron does not change its "shape" if we "zoom in/out", and can be verified analytically by inspecting the above equation. In other words, we have an identity

$$
\theta_{\lambda a}(\lambda a, \lambda b, \lambda c, \lambda d, \lambda e, \lambda f)=\theta_{a}(a, b, c, d, e, f), \quad \lambda>0 .
$$

By differentiating this identity with respect to $\lambda$ and $a, b, c, d, e, f$, it is easy to derive the so-called Schläfli differential identity for a tetrahedron

$$
\sum_{s=1}^{6} l_{s} \frac{\partial \theta_{s}}{\partial l_{p}}=0, \quad \forall l_{p} \in\{a, b, c, d, e, f\} .
$$

As far as the $6 j$ symbols are concerned, we are mainly interested in the asymptotic formulae for large spins. The limit of large spins is defined as a limit when the scaling parameter $\Lambda$ tends to infinity. The relation between a spin and $\Lambda$ is defined by

$$
\lim _{j \rightarrow \infty} f(j) \equiv \lim _{\Lambda \rightarrow \infty} f\left(\Lambda l_{k}-\frac{1}{2}\right), \quad \text { where } \quad l_{k}=k+\frac{1}{2} .
$$

Here $k$ is some initial finite spin, which is scaled to $j$ via $\Lambda$ according to the map

$$
j+\frac{1}{2}=\Lambda\left(k+\frac{1}{2}\right) .
$$


In other words, it is not spins themselves that are being scaled, but rather their corresponding edge lengths.

The large-spin asymptotics of a $6 j$ symbol can be defined in the following way. Fix the 6 initial spins $k_{1}, \ldots, k_{6}$, and associate to them a tetrahedron with the edge lengths $l_{i}=k_{i}+\frac{1}{2}$. This tetrahedron is then scaled by the parameter $\Lambda$ into a new tetrahedron with the edge lengths $\Lambda l_{i}$. Let us denote the corresponding spins as $j_{i}$, then the following asymptotic equation holds $[9,10]$ :

$$
\begin{aligned}
\left\{\begin{array}{ccc}
j_{1} & j_{2} & j_{3} \\
j_{4} & j_{5} & j_{6}
\end{array}\right\} & \equiv\left\{\begin{array}{ccc}
\Lambda l_{1}-\frac{1}{2} & \Lambda l_{2}-\frac{1}{2} & \Lambda l_{3}-\frac{1}{2} \\
\Lambda l_{4}-\frac{1}{2} & \Lambda l_{5}-\frac{1}{2} & \Lambda l_{6}-\frac{1}{2}
\end{array}\right\} \\
& \approx \frac{1}{\sqrt{12 \pi \Lambda^{3} V(l)}} \cos \mathcal{S}(\Lambda, l),
\end{aligned}
$$

where

$$
\mathcal{S}(\Lambda, l)=\Lambda \sum_{s=1}^{6} l_{s} \theta_{s}(l)+\frac{\pi}{4}+\frac{1}{\Lambda} F_{D L}(l)+O\left(\frac{1}{\Lambda^{2}}\right) .
$$

The first term in $\mathcal{S}$ represents the familiar Regge action, while $F_{D L}(l)$ is a very complicated correction of $O(1 / \Lambda)$; see equation (32) of [10].

The second possible configuration is when five spins in a $6 j$ symbol are large, of $O(\Lambda)$, while one is of $O(1)$. In this case, we have the following asymptotic formula:

$$
\left\{\begin{array}{ccc}
j_{1} & j_{2} & j_{3} \\
j_{2}+k_{2} & j_{1}+k_{1} & k_{3}
\end{array}\right\} \approx \frac{(-1)^{j_{1}+j_{2}+j_{3}+k_{2}+k_{3}}}{\sqrt{\left(2 j_{1}+1\right)\left(2 j_{2}+1\right)}} d_{k_{2}, k_{1}}^{\left(k_{3}\right)}(\theta)
$$


where

$$
\cos \theta=\frac{j_{1}\left(j_{1}+1\right)+j_{2}\left(j_{2}+1\right)-j_{3}\left(j_{3}+1\right)}{2 \sqrt{j_{1}\left(j_{1}+1\right) j_{2}\left(j_{2}+1\right)}}, \quad 0 \leqslant \theta \leqslant \pi .
$$

Here $d_{k_{2}, k_{1}}^{\left(k_{3}\right)}(\theta)$ are the usual matrix elements of the $S U(2)$ rotation operator; see [9].

Next we have a configuration with only four large spins, and the corresponding asymptotics is given by

$$
\begin{aligned}
& \left\{\begin{array}{lll}
k_{1} & j+k_{2} & j+k_{3} \\
k_{4} & j+k_{5} & j
\end{array}\right\} \\
& \approx \frac{(-1)^{k_{1}+k_{4}+\min \left(k_{2}+k_{5}, k_{3}\right)}}{\left|k_{2}+k_{5}-k_{3}\right| !}(2 j)^{-1-\left|k_{2}+k_{5}-k_{3}\right|}\left[1+O\left(\frac{1}{j^{2}}\right)\right] \\
& \quad \times\left[\frac{\left(k_{1}-k_{2}+k_{3}\right) !\left(k_{1}-k_{5}\right) !\left(k_{4}-k_{5}+k_{3}\right) !\left(k_{4}-k_{2}\right) !}{\left(k_{1}+k_{2}-k_{3}\right) !\left(k_{1}+k_{5}\right) !\left(k_{4}+k_{5}-k_{3}\right) !\left(k_{4}+k_{2}\right) !}\right]
\end{aligned}
$$

see $[9]$.

Finally, the configuration with only three large spins has the asymptotic formula proportional to the Wigner $3 j$ symbol [9]

$$
\left\{\begin{array}{ccc}
k_{1} & k_{2} & k_{3} \\
j+k_{4} j+k_{5} & j
\end{array}\right\} \approx(-1)^{k_{1}+k_{2}+k_{3}+2\left(k_{4}+k_{5}\right)} \frac{1}{\sqrt{2 j}}\left(\begin{array}{llc}
k_{1} & k_{2} & k_{3} \\
k_{5}-k_{4} & k_{4}-k_{5}
\end{array}\right) .
$$

As a final remark, note that the spins and the corresponding edge lengths for a $6 j$ symbol are constrained by the triangle inequalities. However, these constraints are not sufficient to guarantee the condition $V^{2}>0$ for the volume of the $6 j$ symbol tetrahedron. There are choices of spins such that the $6 j$ symbol is well defined, and the left-hand side of (B.3) is real, while $V^{2}<0$. Consequently the right-hand side of (B.3) is a complex number. Of course, in such situations the equation (B.3) does not apply, and we have a different, exponentially decreasing asymptotics; see [9]. However, we are interested in the extremal points of the asymptotic formula, and the exponentially decreasing asymptotics does not have any such points, so it is not relevant for our purposes. 


\section{Appendix C The cosine approximation}

In this section, we shall give a rigorous derivation of the "cosine formula" (4.5). We start by introducing the Heaviside step-function as

$$
H(x)= \begin{cases}0, & \text { for } x<0 \\ \frac{1}{2}, & \text { for } x=0 \\ 1, & \text { for } x>0\end{cases}
$$

This is a standard definition, and the standard rules apply. For example, derivative of a Heaviside function is the Dirac $\delta$ function and the integral of $\delta$-function from $-\infty$ to $x$ gives $H(x)$. We can then define the so-called rectangle function

$$
K_{a}(x) \equiv H(x+a) H(-x+a)= \begin{cases}0, & \text { for } x<-a, \\ \frac{1}{2}, & \text { for } x=-a \\ 1, & \text { for }-a<x<a \\ \frac{1}{2}, & \text { for } x=a, \\ 0, & \text { for } x>a .\end{cases}
$$

The function $K_{a}(x)$ is equal to one in the interval $(-a, a)$, zero outside, and at the points $-a$ and $a K$ is conveniently defined to be $\frac{1}{2}$, so that it has a nice property proven in Lemma C.1.

Lemma C.1. The following identities hold

$$
\begin{gathered}
K_{b}(x+(a+b))+K_{a}(x)+K_{b}(x-(a+b))=K_{a+2 b}(x), \\
\sum_{k \in \mathbb{Z}} K_{a}(x-2 k a)=1 .
\end{gathered}
$$

Proof. The first identity can be demonstrated by using the definition of $K$. First, we see that outside of the interval $[-a-2 b, a+2 b]$ both the left-hand and the right-hand sides of (C.1) are equal to zero. Next, the three terms on the left-hand side of (C.1) are equal to one respectively in the intervals $(-a-2 b,-a),(-a, a)$ and $(a, a+2 b)$. Finally, at points $-a$ and $a$ the appropriate terms are equal to $\frac{1}{2}$ and add up to one so that the resulting function is continuous and equal to one in the whole interval $(-a-2 b, a+2 b)$. At the boundary points of $[-a-2 b, a+2 b]$, the left-hand 
side of (C.1) gives the contribution of $\frac{1}{2}$. All these results taken together form by definition the right-hand side of (C.1).

The identity (C.2) can be proved by applying the identity (C.1) iteratively. First note that the sum over all integers is in fact defined as the limit $m \rightarrow \infty$ of the sum over the domain $-m,-m+1, \ldots, m-1, m$. Consequently we can write:

$$
\sum_{k \in \mathbb{Z}} K_{a}(x-2 k a)=\lim _{m \rightarrow \infty} \sum_{k=-m}^{m} K_{a}(x-2 k a) .
$$

Now note that by applying the first identity $m$ times we have

$$
\sum_{k=-m}^{m} K_{a}(x-2 k a)=K_{(2 m+1) a}(x) .
$$

This identity can be also easily seen graphically, and can be proved by induction over $m$. Therefore,

$$
\sum_{k \in \mathbb{Z}} K_{a}(x-2 k a)=\lim _{m \rightarrow \infty} K_{(2 m+1) a}(x)=K_{\infty}(x)=1 .
$$

The function $K_{a}(x)$ was introduced because it allows for a neat "cutting" of the appropriate pieces of the real line. Now we make use of this property in order to prove the following identity.

Lemma C.2. The identity

$$
\cos x=\sum_{k \in \mathbb{Z}}(-1)^{k} K_{\frac{\pi}{2}}(x-k \pi) \cos (x-k \pi)
$$

holds.

Proof. Start from the right-hand side and compute the left-hand side in the following way

$$
\begin{aligned}
\mathrm{RHS} & =\sum_{k \in \mathbb{Z}}(-1)^{k} K_{\frac{\pi}{2}}(x-k \pi)(\cos x \cos k \pi+\sin x \sin k \pi) \\
& =\sum_{k \in \mathbb{Z}}(-1)^{k} K_{\frac{\pi}{2}}(x-k \pi)(-1)^{k} \cos x \\
& =\cos x \sum_{k \in \mathbb{Z}} K_{\frac{\pi}{2}}(x-k \pi) \\
& =\cos x .
\end{aligned}
$$


Now if we note that $K_{\frac{\pi}{2}}(x-k \pi) \cos (x-k \pi)$ is continuous and nonnegative for all $x \in \mathbb{R}$ and $k \in \mathbb{Z}$, the statement of Lemma C.2 can be rewritten in the form

$$
\cos x=\sum_{k \in \mathbb{Z}}(-1)^{k} \mathrm{e}^{\ln \left[K_{\frac{\pi}{2}}(x-k \pi) \cos (x-k \pi)\right]},
$$

which represents the key "cosine formula". The exponent can be expanded into a power series around the point $x=k \pi$,

$$
\begin{aligned}
\ln \left[K_{\frac{\pi}{2}}(x-k \pi) \cos (x-k \pi)\right]= & -\frac{1}{2}(x-k \pi)^{2}-\frac{1}{12}(x-k \pi)^{4} \\
& +O(x-k \pi)^{6}, \quad(x \rightarrow k \pi),
\end{aligned}
$$

and in the leading order approximation we can write

$$
\cos x \approx \sum_{k \in \mathbb{Z}}(-1)^{k} \mathrm{e}^{-\frac{1}{2}(x-k \pi)^{2}+R(x, k)},
$$

where $R(x, k)$ is the remainder of $O\left((x-k \pi)^{4}\right)$ when $x \rightarrow k \pi$.

This kind of approximation is useful since the cosine function is well approximated in the vicinity of all extremal points simultaneously. In the crudest approximation, the remainder $R(x, k)$ can be substituted by some average value over one period of the cosine, $\bar{R}$, so we write:

$$
\cos x \approx \mathrm{e}^{\bar{R}} \sum_{k \in \mathbb{Z}}(-1)^{k} \mathrm{e}^{-\frac{1}{2}(x-k \pi)^{2}}, \quad x \rightarrow k \pi .
$$

The constant $\bar{R}$ can be calculated, because at any point $x=k_{0} \pi\left(k_{0} \in \mathbb{Z}\right)$ the formula must become exact, with no error. Namely, expanding the exact exponent into a power series and discarding certain terms we effectively allow for the appearance of the "tails" in each Gaussian in the sum. These tails then give artificial contribution to other Gaussians, and the constant $e^{\bar{R}}$ accounts for the appropriate "correction". For example, substituting $x=0$ into the left and right side, we have

$$
1=\mathrm{e}^{\bar{R}} \sum_{k \in \mathbb{Z}}(-1)^{k} \mathrm{e}^{\frac{\pi^{2}}{2} k^{2}},
$$

so that

$$
e^{\bar{R}}=\frac{1}{\vartheta_{4}\left(0, \mathrm{e}^{-\frac{\pi^{2}}{2}}\right)} \approx 1,01459 .
$$

Here $\vartheta_{4}(u, q)$ is the so-called inverse elliptic theta-function of the fourth kind. Of course, if we had kept the $x^{4}$ term in the exponent, the Gaussian 
tails would have been different and the constant $\bar{R}$ would have had different numerical value, but again such that the equation is exact at all points $x=k \pi$. If we had kept all terms in the exponent, the tails would have vanished and this constant would have been equal to one.

Anyway, we are interested only in the crudest approximation of the cosine function with Gaussian functions, so that the formula we will use is

$$
\cos x \approx \frac{1}{\vartheta_{4}\left(0, e^{-\frac{\pi^{2}}{2}}\right)} \sum_{k \in \mathbb{Z}}(-1)^{k} \mathrm{e}^{-\frac{1}{2}(x-k \pi)^{2}}, \quad x \rightarrow k \pi .
$$

One very important remark here is that this equation makes sense only in the vicinity of cosine extremal points, i.e., in the neighborhood of the points $x=k \pi$. Therefore, one should make sure that the argument of the cosine is close enough to some integer multiple of $\pi$, whenever one applies the formula. For the points in the vicinity of $k \pi / 2$, the approximation is less accurate.

\section{Appendix D Laplace's method}

Let $f(x)$ be a real function in the interval $[a, b]$ such that it has a single global maximum at a point $x_{0} \in[a, b]$. We would like to find the asymptotics of the integral

$$
I=\int_{a}^{b} d x \mathrm{e}^{\Lambda f(x)}
$$

for large $\Lambda$. We will describe here the Laplace method of calculating this asymptotics.

The idea of the method is the fact that $\mathrm{e}^{\Lambda f(x)}$ goes to infinity at the fastest rate at the point of the global maximum as $\Lambda \rightarrow \infty$. One can then approximate $\mathrm{e}^{\Lambda f(x)}$ in $[a, b]$ by a Gaussian function centered at $x_{0}$. This also implies that the integration domain $[a, b]$ can be extended to the whole real line, since the "tails" of a Gaussian function do not give a significant contribution. By using the Taylor series for $f(x)$ at the point $x_{0}$ we obtain

$$
f(x)=\sum_{n=0}^{\infty} \frac{f^{(n)}\left(x_{0}\right)}{n !}\left(x-x_{0}\right)^{n}=f\left(x_{0}\right)-\frac{1}{2}\left|f^{\prime \prime}\left(x_{0}\right)\right|\left(x-x_{0}\right)^{2}+O\left(x-x_{0}\right)^{3} .
$$


By changing the integration variable to $y=x-x_{0}$, the integral $I$ is reduced to a sum of Gaussian integrals

$$
I=\mathrm{e}^{\Lambda f\left(x_{0}\right)} \int_{\mathbb{R}} d y \mathrm{e}^{-\frac{\Lambda}{2}\left|f^{\prime \prime}\left(x_{0}\right)\right| y^{2}}\left(\sum_{n=0}^{\infty} \frac{\Lambda^{n}}{n !}\left[O\left(y^{3}\right)\right]^{n}\right),
$$

which implies

$$
I=\mathrm{e}^{\Lambda f\left(x_{0}\right)} \sqrt{\frac{2 \pi}{\Lambda\left|f^{\prime \prime}\left(x_{0}\right)\right|}}\left[1+O\left(\frac{1}{\Lambda}\right)\right] .
$$

In the case when there are several global maxima in $[a, b]$ with the same value of $f(x)$, then the formula (D.1) becomes

$$
I=\sum_{x_{0} \in[a, b]} \mathrm{e}^{\Lambda f\left(x_{0}\right)} \sqrt{\frac{2 \pi}{\Lambda\left|f^{\prime \prime}\left(x_{0}\right)\right|}}\left[1+O\left(\frac{1}{\Lambda}\right)\right] .
$$

\section{Appendix E Generalized Gaussian integrals}

Let us start from the identity

$$
\int_{\mathbb{R}} d x \mathrm{e}^{-\frac{1}{2} a x^{2}+b x}=\sqrt{\frac{2 \pi}{a}} \mathrm{e}^{\frac{b^{2}}{2 a}}, \quad a>0 .
$$

This identity can be generalized to $\mathbb{R}^{n}$

$$
\int_{\mathbb{R}^{n}} d^{n} x \mathrm{e}^{-\frac{1}{2} x^{\mathrm{T}} A x+B x}=\sqrt{\frac{(2 \pi)^{n}}{\operatorname{det} A}} \mathrm{e}^{\frac{1}{2} B A B^{\mathrm{T}}}
$$

where $A$ and $B$ are matrices of the type $n \times n$ and $1 \times n$ respectively and $A$ is a symmetric matrix. This identity can be derived by reducing the integral in (E.1) to a product of one-dimensional integrals by a change of variables $x^{\prime}=O x$, where $O$ is the orthogonal matrix which diagonalizes the matrix $A$. In the diagonal basis, the integral reduces to a product of $n$ one-dimensional integrals. The critical assumption here is that the eigenvalues of $A$ must be strictly positive if the integral is to converge. However, we are interested in the situation when some of the eigenvalues of $A$ are zero. In this case, the integral in (E.1) diverges, and our goal now is to regularize this divergence in one special case. 
Let us start with an integral over a compact domain $D=[-G, G]^{n}$

$$
I_{D}=\int_{D} d^{n} x \mathrm{e}^{-\frac{1}{2} x^{\mathrm{T}} A x+B x} .
$$

For simplicity, assume that the matrix $A$ is already diagonal, and denote its rank as $r<n$. Also, denote all $n$ eigenvalues as $a_{i}$, and let $a_{1}, \ldots, a_{r} \neq 0$, while $a_{r+1}, \ldots, a_{n}=0$. Next, let $K$ be the projector to the null-space of matrix $A$, and assume that $B K=0$. This means that in this particular basis we have $B_{r+1}, \ldots, B_{n}=0$. Now the integral $I_{D}$ can be split into a product of $r$ Gaussian one-dimensional integrals and $n-r$ one-dimensional integrals of a constant function, where the constant is equal to one,

$$
I_{D}=\prod_{i=1}^{r} \int_{-G}^{G} d x_{i} \mathrm{e}^{-\frac{1}{2} x_{i}^{2} a_{i}+B_{i} x_{i}} \prod_{i=r+1}^{n} \int_{-G}^{G} d x_{i} \underbrace{\mathrm{e}^{-\frac{1}{2} x_{i}^{2} a_{i}+B_{i} x_{i}}}_{1} .
$$

In the limit $G \rightarrow \infty$ we will define a regularized $I$

$$
I=\frac{\lim _{G \rightarrow \infty} I_{D}}{\lim _{G \rightarrow \infty}\left[\int_{-G}^{G} d x\right]^{n-r}}=\sqrt{\frac{(2 \pi)^{r}}{\operatorname{det} M}} \mathrm{e}^{\frac{1}{2} N M^{-1} N^{\mathrm{T}}} .
$$

Here $M$ and $N$ denote the submatrices of $A$ and $B$ of type $r \times r$ and $1 \times r$, which are obtained by simultaneous change of basis which puts $A$ and $B$ in block-diagonal form:

$$
A=\left[\begin{array}{cc}
M & 0 \\
0 & 0
\end{array}\right], \quad B=\left[\begin{array}{ll}
N & 0
\end{array}\right]
$$

Simultaneous diagonalization is possible because of the imposed assumption $B K=0$.

\section{Appendix F Matrix theorems}

Here we explain some results for matrices that we have used in the main text. These results can be found in [11]. However, one of the results, the statement (c) bellow, is a new result, to the best of our knowledge. 
Theorem F.1. Let $\Delta$ be a symmetric real matrix of type $n \times n$ and let $R$ be its rank. Let us split $\Delta$ into blocks as

$$
\Delta=\left[\begin{array}{cc}
S & N \\
N^{T} & M
\end{array}\right]
$$

where $S$ is a $J \times J$ matrix, $N$ is a $J \times r$ matrix, $M$ is a $r \times r$ matrix and $n=J+r$. We will also assume that $M$ is invertible.

Let us construct the Schur complement (see [11]) $\tilde{S}$, which is a $J \times J$ matrix

$$
\tilde{S}=S-N M^{-1} N^{T}
$$

Denote the rank of $\tilde{S}$ as $\rho$. Then

(a) $R=r+\rho$ (Guttman rank additivity);

(b) $\operatorname{det} \Delta=\operatorname{det} \tilde{S} \operatorname{det} M$ (Schur determinant formula);

(c) if $0<\rho<J$, then

$$
\operatorname{det} M_{\Delta}\left(\operatorname{det} B_{4}\right)^{2}=\operatorname{det} M \operatorname{det} M_{\tilde{S}}
$$

Here $M_{\Delta}$ and $M_{\tilde{S}}$ are invertible $R \times R$ and $\rho \times \rho$ matrices, respectively. They are obtained by using orthogonal transformations which put $\Delta$ and $\tilde{S}$ into a block-diagonal form

$$
\Delta=\left[\begin{array}{cc}
0 & 0 \\
0 & M_{\Delta}
\end{array}\right], \quad \tilde{S}=\left[\begin{array}{cc}
0 & 0 \\
0 & M_{\tilde{S}}
\end{array}\right]
$$

The $B_{4}$ matrix will be explicitly constructed in the proof below.

Proof. We start from the Aitken block diagonalization formula [11] and from now on we use $I$ to denote a unit matrix of any size appropriate for its position in an equation

$$
\left[\begin{array}{cc}
I & -N M^{-1} \\
0 & I
\end{array}\right]\left[\begin{array}{cc}
S & N \\
N^{T} & M
\end{array}\right]\left[\begin{array}{cc}
I & 0 \\
-M^{-1} N^{T} & I
\end{array}\right]=\left[\begin{array}{cc}
\tilde{S} & 0 \\
0 & M
\end{array}\right]
$$

This equation can be verified by a direct multiplication of the left-hand side. Denoting the first matrix on the left as $A$, we can rewrite this identity in a compact form $A \Delta A^{\mathrm{T}}=\tilde{S} \oplus M$. The rank of the right-hand side is the sum of ranks of $\tilde{S}$ and $M$, which amounts to $\rho+r$. Since the rank of $A$ is 
equal to its dimension $n$, the total rank of the product on the left-hand side is equal to the rank of $\Delta$, so we easily obtain

$$
R=r+\rho,
$$

which completes the proof of part (a).

Next, we take the determinant of (F.2). Since $A$ is block-triangular, its determinant is a product of determinants of blocks on the diagonal, so that we obtain $\operatorname{det} A=1$. The left-hand side is thus the product of determinants, $\operatorname{det} A \operatorname{det} \Delta \operatorname{det} A^{\mathrm{T}}$, and it is equal to $\operatorname{det} \Delta$ because $\operatorname{det} A^{T}=\operatorname{det} A=1$. On the right-hand side we have a block-diagonal matrix, so that its determinant is equal to $\operatorname{det} \tilde{S} \operatorname{det} M$. Hence,

$$
\operatorname{det} \Delta=\operatorname{det} \tilde{S} \operatorname{det} M,
$$

which completes the proof of part (b).

In order to prove (c), let $O$ be a $J \times J$ orthogonal matrix, which transforms $\tilde{S}$ into a block-reduced form,

$$
O \tilde{S} O^{\mathrm{T}}=0 \oplus M_{\tilde{S}}
$$

Since $\rho \neq 0$, matrix $\tilde{S}$ has exactly $\rho$ non-zero eigenvalues, which constitute $M_{\tilde{S}}$, and since $\tilde{S}$ is also real and symmetric, there will always exist an orthogonal matrix $O$ that diagonalizes it. Given that the eigenvalues of $M_{\tilde{S}}$ are non-zero, it is invertible. The zero-block is of type $\nu \times \nu$, where $\nu=J-\rho$ is the dimension of the null-space of $\tilde{S}$. By using $O$, one can construct an orthogonal $n \times n$ matrix $P=O \oplus I$ such that

$$
P(\tilde{S} \oplus M) P^{\mathrm{T}}=0 \oplus M_{\tilde{S}} \oplus M .
$$

By using an analogous argument one can always construct an orthogonal $n \times n$ matrix $Q^{\mathrm{T}}$ such that

$$
Q^{\mathrm{T}} \Delta Q=0 \oplus M_{\Delta}, \quad \Leftrightarrow \quad \Delta=Q\left(0 \oplus M_{\Delta}\right) Q^{\mathrm{T}} .
$$

The zero block comes from the null-space of $\Delta$. It is of the size $n-R$, which is also equal to $\nu$, since $n=J+r$ and $R=r+\rho$ according to the part (a) of Theorem F.1. 
Consider (F.2), and multiply it by $P$ from the left and by $P^{\mathrm{T}}$ from the right, and use (F.3) and (F.4) to rewrite it in the form

$$
P A Q\left(0 \oplus M_{\Delta}\right) Q^{\mathrm{T}} A^{\mathrm{T}} P^{\mathrm{T}}=0 \oplus M_{\tilde{S}} \oplus M .
$$

Let us introduce the matrix $B \equiv P A Q$ and write it in the block form as

$$
B=\left[\begin{array}{ll}
B_{1} & B_{2} \\
B_{3} & B_{4}
\end{array}\right]
$$

where the blocks $B_{1}, B_{2}, B_{3}$ and $B_{4}$ are $\nu \times \nu, \nu \times R, R \times \nu$ and $R \times R$ matrices, respectively. Substituting this into the left-hand side of (F.5) yields

$$
P A Q\left(0 \oplus M_{\Delta}\right) Q^{\mathrm{T}} A^{\mathrm{T}} P^{\mathrm{T}} \equiv B\left[\begin{array}{cc}
0 & 0 \\
0 & M_{\Delta}
\end{array}\right] B^{\mathrm{T}}=\left[\begin{array}{cc}
B_{2} M_{\Delta} B_{2}^{\mathrm{T}} & B_{2} M_{\Delta} B_{4}^{\mathrm{T}} \\
B_{4} M_{\Delta} B_{2}^{\mathrm{T}} & B_{4} M_{\Delta} B_{4}^{\mathrm{T}}
\end{array}\right] .
$$

By comparing (F.6) to the right-hand side of (F.5), we obtain

$$
\left[\begin{array}{ll}
B_{2} M_{\Delta} B_{2}^{\mathrm{T}} & B_{2} M_{\Delta} B_{4}^{\mathrm{T}} \\
B_{4} M_{\Delta} B_{2}^{\mathrm{T}} & B_{4} M_{\Delta} B_{4}^{\mathrm{T}}
\end{array}\right]=\left[\begin{array}{ccc}
0 & 0 & 0 \\
0 & M_{\tilde{S}} & 0 \\
0 & 0 & M
\end{array}\right]
$$

Note that the zero-block of (F.7) is a $\nu \times \nu$ matrix, which is also the $B_{2} M_{\Delta} B_{2}^{\mathrm{T}}$ block. We then read off the following equations

$$
\begin{gathered}
B_{4} M_{\Delta} B_{4}^{\mathrm{T}}=M_{\tilde{S}} \oplus M, \\
B_{2} M_{\Delta} B_{4}^{\mathrm{T}}=0, \\
B_{2} M_{\Delta} B_{2}^{\mathrm{T}}=0 .
\end{gathered}
$$

By taking the determinant of (F.8), we finally obtain

$$
\operatorname{det} M_{\Delta}\left(\operatorname{det} B_{4}\right)^{2}=\operatorname{det} M \operatorname{det} M_{\tilde{S}}
$$

This establishes (F.1) and completes the proof of part (c) of the theorem.

Given that $M, M_{\tilde{S}}$ and $M_{\Delta}$ are all invertible, we have $\operatorname{det} B_{4} \neq 0$ which means that $B_{4}$ is also invertible. By multiplying (F.9) by $\left(B_{4}^{\mathrm{T}}\right)^{-1} M_{\Delta}^{-1}$ from 
the right, we obtain

$$
B_{2}=0 .
$$

The equation (F.10) now vanishes and does not provide any additional constraint. Therefore, the matrix $B$ has the following form

$$
B \equiv P A Q=\left[\begin{array}{cc}
B_{1} & 0 \\
B_{3} & B_{4}
\end{array}\right]
$$

Remark F.1. The $\Delta$ matrix from the main text has the form

$$
\Delta=\left[\begin{array}{ccc}
S & N & 0 \\
N^{\mathrm{T}} & M & 0 \\
0 & 0 & 0
\end{array}\right]
$$

which differs from the one in Theorem F.1 by an additional zero-block of size $\Omega-n$. However, these additional zeroes are integrated out before the Theorem F.1 is applied, and they do not affect the statements of Theorem F.1.

Remark F.2. The result (c) is a generalization of the result (b) to the case when $\Delta$ is a singular matrix. While the part (b) is in fact valid for singular matrices, it merely states that $0=0$ and provides no information about non-singular principal minors of $\Delta$. The result (c) is more fine-grained, and provides precisely this non-trivial information about $\Delta$.

It was assumed in the part (c) that $0<\rho<J$. If $\rho=J$ then $\Delta$ is a regular matrix, and hence the result (b) can be used. If $\rho=0$, then $\tilde{S}=0, \nu=J$, and instead of (F.8) we obtain

$$
B_{4} M_{\Delta} B_{4}^{\mathrm{T}}=M,
$$

and consequently

$$
\operatorname{det} M_{\Delta}\left(\operatorname{det} B_{4}\right)^{2}=\operatorname{det} M \text {. }
$$

In this case we can set $P=I$ and obtain

$$
B \equiv A Q=\left[\begin{array}{cc}
B_{1} & 0 \\
B_{3} & B_{4}
\end{array}\right]
$$

for the matrix $B$.

Remark F.3. In the main text, we use the results (b) and (c) to determine the leading $\Lambda$-order of the Schur complement $\tilde{S}$, knowing that $\Delta$ is of $O(1)$. 
However, it is necessary to show that $B_{4}$ is of $O(1)$ as well. In order to do this, note that

$$
\operatorname{det} B=\operatorname{det} P \operatorname{det} A \operatorname{det} Q= \pm 1,
$$

since $P$ and $Q$ are unitary matrices. On the other hand, from (F.11) we know that $\operatorname{det} B=\operatorname{det} B_{1} \operatorname{det} B_{4}$, so that we have

$$
\operatorname{det} B_{1} \operatorname{det} B_{4}= \pm 1 \text {. }
$$

Let us now assume that the blocks $B_{1}$ and $B_{4}$ are of order $k$ and $m$ in $1 / \Lambda$, respectively

$$
B_{1}=\frac{C}{\Lambda^{k}}+O\left(\frac{1}{\Lambda^{k+1}}\right), B_{4}=\frac{D}{\Lambda^{m}}+O\left(\frac{1}{\Lambda^{m+1}}\right), \quad k, m \geqslant 0, C, D \sim O(1) .
$$

The numbers $k$ and $m$ cannot be negative since the whole $B$ matrix must be of $O(1)$. Namely, the matrices $P$ and $Q$ are orthogonal, and consequently all their elements are bounded above by 1 . Thus $P$ and $Q$ are of $O(1)$. The matrix $A$ is also of $O(1)$, since $\Delta$ and consequently $M, N, M^{-1}$ are all of the same order. Therefore, $B=P A Q \sim O(1)$.

Since $B_{1}$ is a $\nu \times \nu$ matrix and $B_{4}$ is a $R \times R$ matrix, then $\operatorname{det} B_{1}=\frac{1}{\Lambda^{k \nu}} \operatorname{det} C+O\left(\frac{1}{\Lambda^{k+1}}\right), \quad \operatorname{det} B_{4}=\frac{1}{\Lambda^{m R}} \operatorname{det} D+O\left(\frac{1}{\Lambda^{m+1}}\right)$.

By substituting (F.13) back into (F.12) we obtain the consistency equation

$$
k \nu+m R=0 .
$$

Since both $\nu, R>0$ while $k, m \geqslant 0$, the only solution of this equation is $k=m=0$. Therefore

$$
\operatorname{det} B_{4} \sim B_{4} \sim O(1) .
$$

In the case when $\nu=0$ the $\Delta$ matrix is regular and instead of the part (c) we use the part (b) of Theorem F.1. However, the part (b) does not involve $\operatorname{det} B_{4}$, so that we need the above result only for $\nu>0$.

\section{References}

[1] C. Rovelli, Quantum gravity, Cambridge University Press, 2004.

[2] A. Miković, Quantum gravity vacuum and invariants of embedded spin networks, Class. Quant. Grav. 20 (2003), 3483. 
[3] A. Miković, Flat spacetime vacuum in loop quantum gravity, Class. Quant. Grav. 21 (2004), 3909; Class. Quant. Grav. 23 (2006), 5459 (Errata).

[4] J. F. Martins and A. Miković, Invariants of spin networks embedded in three-manifolds, Commun. Math. Phys. 279 (2008), 381.

[5] A. Miković, Spin network wavefunction and nonperturbative graviton propagator, Fortschr. Phys. 56 (2008), 475.

[6] C. Rovelli, Graviton propagator from background-independent quantum gravity, Phys. Rev. Lett. 97 (2006), 151301.

[7] E. Bianchi, L. Modesto, C. Rovelli and S. Speziale, Graviton propagator in loop quantum gravity, Class. Quant. Grav. 23 (2006), 6989.

[8] V. Turaev, Quantum Invariants of knots and three-manifolds, de Gruyter Studies in Mathematics, 18, Walter de Gruyter \& Co., Berlin, 1994.

[9] G. Ponzano and T. Regge, Semiclassical limit of Racah coefficients, in 'Spectroscopic and Group Theoretical Methods in Physics', ed F. Block, North-Holland, Amsterdam, 1968.

[10] M. Dupuis and E. Livine, Pushing further the asymptotics of the 6jsymbol, Phys. Rev. D 80 (2009), 024035.

[11] F. Zhang, The Schur complement and its applications, Springer, New York, 2005.

[12] J. Engle, R. Pereira and C. Rovelli, The loop-quantum-gravity vertexamplitude, Phys. Rev. Lett. 99 (2007), 161301.

[13] J. Engle, E. Livine, R. Pereira and C. Rovelli, $L Q G$ vertex with finite Immirzi parameter, Nucl. Phys. B 799 (2008), 136-149.

[14] L. Freidel and K. Krasnov, A new spin foam model for $4 d$ gravity, Class. Quant. Grav. 25 (2008), 125018.

[15] L. Freidel and D. Louapre, Diffeomorphisms and spin foam models, Nucl. Phys. B 662 (2003), 279-298.

[16] J.S. Catrer, D.E. Flath and M. Saito, The classical and quantum 6jsymbols, Princeton University Press, New Jersey, 1995. 
